\title{
Chemogenetic stimulation of striatal projection neurons modulates responses to Parkinson's disease therapy
}

\author{
Cristina Alcacer, ${ }^{1}$ Laura Andreoli, ${ }^{1}$ Irene Sebastianutto, ${ }^{1}$ Johan Jakobsson, ${ }^{2}$ Tim Fieblinger, ${ }^{1}$ and Maria Angela Cenci ${ }^{1}$ \\ 'Basal Ganglia Pathophysiology Unit, Department of Experimental Medical Science, Lund University, Lund, Sweden. ${ }^{2}$ Laboratory of Molecular Neurogenetics, Wallenberg Neuroscience Center, \\ Lund University, Lund, Sweden.
}

\begin{abstract}
Parkinson's disease (PD) patients experience loss of normal motor function (hypokinesia), but can develop uncontrollable movements known as dyskinesia upon treatment with L-DOPA. Poverty or excess of movement in PD has been attributed to overactivity of striatal projection neurons forming either the indirect (iSPNs) or the direct (dSPNs) pathway, respectively. Here, we investigated the two pathways' contribution to different motor features using SPN type-specific chemogenetic stimulation in rodent models of PD (PD mice) and L-DOPA-induced dyskinesia (LID mice). Using the activatory Gq-coupled human M3 muscarinic receptor (hM3Dq), we found that chemogenetic stimulation of dSPNs mimicked, while stimulation of iSPNs abolished the therapeutic action of L-DOPA in PD mice. In LID mice, hM3Dq stimulation of dSPNs exacerbated dyskinetic responses to L-DOPA, while stimulation of iSPNs inhibited these responses. In the absence of L-DOPA, only chemogenetic stimulation of dSPNs mediated through the Gs-coupled modified rat muscarinic M3 receptor (rM3Ds) induced appreciable dyskinesia in PD mice. Combining D2 receptor agonist treatment with rM3Ds-dSPN stimulation reproduced all symptoms of LID. These results demonstrate that dSPNs and iSPNs oppositely modulate both therapeutic and dyskinetic responses to dopamine replacement therapy in PD. We also show that chemogenetic stimulation of different signaling pathways in dSPNs leads to markedly different motor outcomes. Our findings have important implications for the design of effective antiparkinsonian and antidyskinetic drug therapies.
\end{abstract}

\section{Introduction}

Our capacity to perform well-coordinated movements depends on the activity of 2 pathways linking the striatum with deep basal ganglia nuclei. The direct pathway originates from striatal neurons projecting monosynaptically to the output nuclei of the basal ganglia (i.e., the globus pallidus pars interna and the substantia nigra pars reticu-

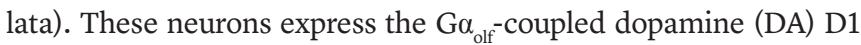
receptor. The indirect pathway originates from neurons projecting to the globus pallidus pars externa, which express the Gai-coupled DA D2 receptor (for review, see ref. 1). Direct and indirect pathway neurons jointly provide the backbone through which nigrostriatal dopaminergic afferents modulate both the point-by-point activity and the long-term plasticity of basal ganglia-thalamocortical circuits $(2-5)$. Seminal studies posited that striatal projection neurons forming the direct and indirect pathways (hereafter referred to as dSPNs and iSPNs, respectively) have an antagonistic role in motor control, selecting and inhibiting movements in a sequence, respectively $(6,7)$. This original prediction was later confirmed by optogenetic stimulation studies in freely moving mice (8). However, the degree of functional segregation between dSPNs and iSPNs is being questioned because direct and indirect pathways interact at striatal and pallidal levels via bridging collaterals and interneuronal synaptic circuits $(9,10)$. Moreover, studies based on cell type-specific

Conflict of interest: The authors have declared that no conflict of interest exists. Submitted: August 12, 2016; Accepted: November 22, 2016.

Reference information: J Clin Invest. 2017;127(2):720-734.

https://doi.org/10.1172/JCI90132. recording techniques have suggested that coactivation of dSPNs and iSPNs is necessary for movement to be produced $(2,11)$. There is, therefore, a need to further clarify the relative roles of these 2 neuronal systems in both normal and pathological movements. As to the latter, most extrapyramidal movement disorders involve some form of striatal dysfunction $(6,7)$, and dissecting the causal contribution of dSPNs and iSPNs to these conditions would greatly facilitate a rational therapeutic development.

The most common disorder of basal ganglia origin is Parkinson's disease (PD), a neurodegenerative condition characterized by a severe degeneration of nigral DA neurons. The ensuing striatal DA depletion causes poverty and slowness of movement (hypokinesia and bradykinesia, respectively) (reviewed in ref. 12). These clinical features are treated with the DA precursor L-DOPA, which restores movement velocity and vigor $(13,14)$. However, the majority of PD patients eventually develop involuntary movements having both hyperkinetic and dystonic components (dyskinesia) (reviewed in ref. 15). L-DOPA-induced dyskinesia (LID) is a debilitating and treatment-limiting condition, affecting up to $80 \%$ of PD patients within 10 years (15). A better understanding of the neural mechanisms that form the basis of LID is essential for designing effective therapeutic strategies $(16,17)$.

In the present study, we have taken advantage of cell typespecific chemogenetics to investigate the role of dSPNs and iSPNs in models of parkinsonism and LID. Our results reveal that direct and indirect pathways have opposing effects on whole-body movements and that they oppositely modulate 

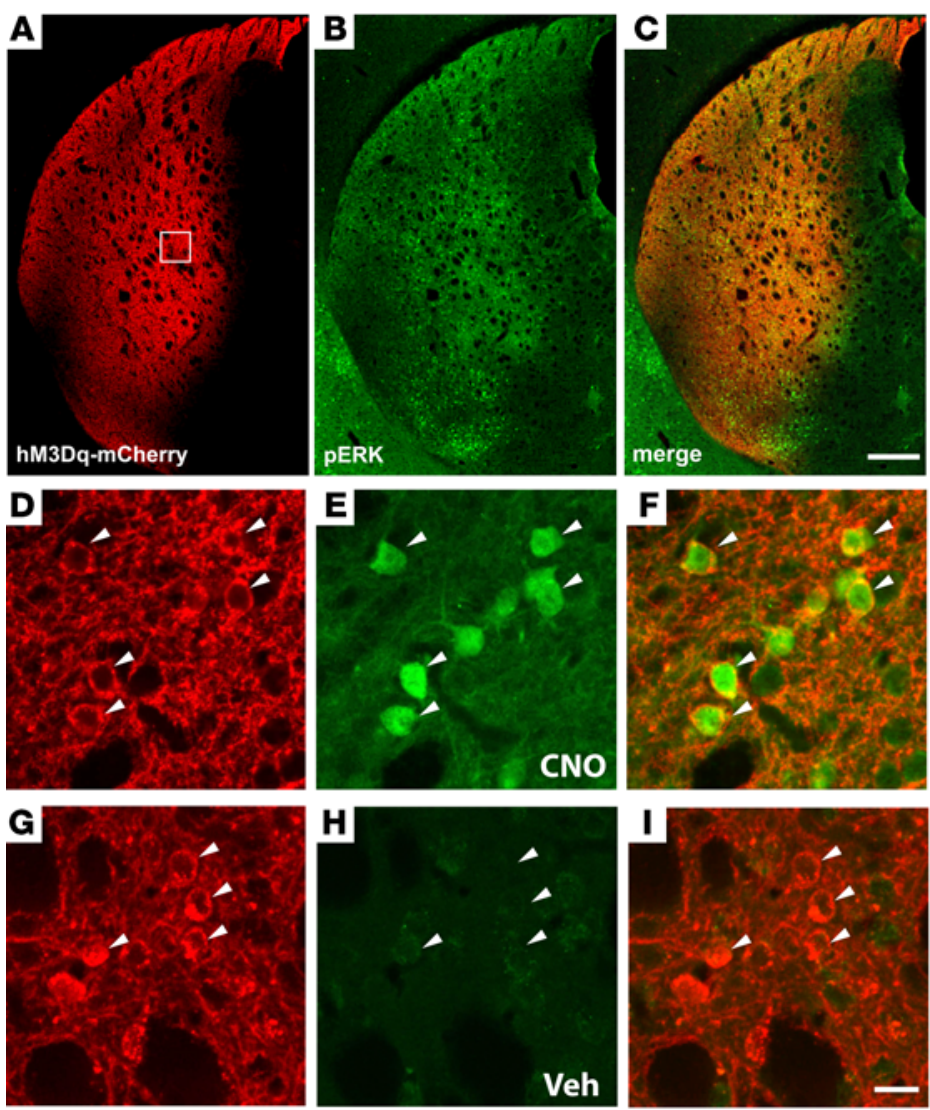

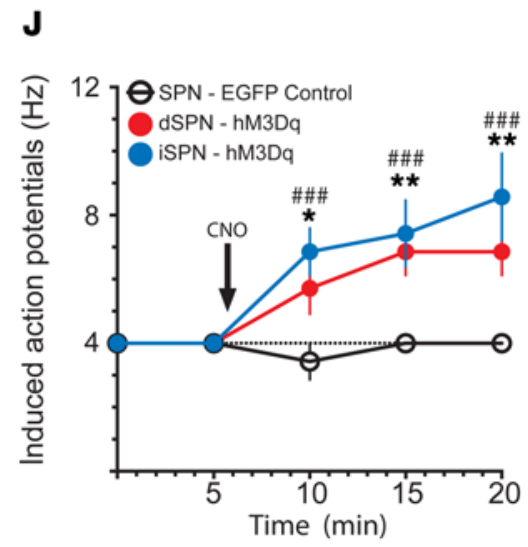

$\mathbf{K}$

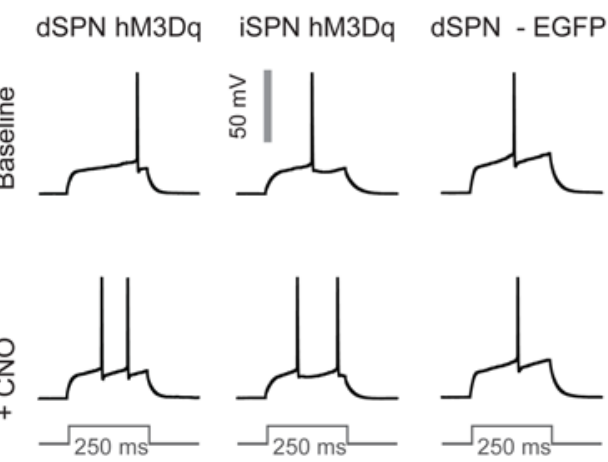

Figure 1. Histomolecular and electrophysiological validation of hM3Dq. (A-I) Photomicrographs were acquired from A2a-Cre transgenic mice injected intrastriatally with the AAV5-hSyn-DIO-hM3Dq-mCherry vector. (A) Low-magnification photograph shows mCherry expression (revealed with RFP antibody) throughout the caudate-putamen (dorsolateral striatum). (B) The transduced striatal region shows positive immunostaining for $\mathrm{p}$-ERK after treatment with CNO (1 mg/kg). (C) Merged confocal photograph demonstrates regional colocalization of hM3Dq-mCherry and p-ERK. (D) High-magnification photomicrograph of a transduced area (cf. inset in A) reveals mCherry immunoreactivity in both neuropile and SPN somas (indicated by arrows). (E) p-ERK immunoreactivity in transduced SPNs after treatment with CNO. (F) Cellular colocalization of the 2 markers demonstrated by the merged confocal picture. (G-I) No p-ERK immunostaining was observed in transduced SPNs after vehicle treatment. Scale bars: $400 \mu \mathrm{m}$ (A-C); $20 \mu \mathrm{m}$ (D-I). (J and K) Electrophysiological response to CNO in hM3Dq- and EGFP-transduced dSPN and iSPN from D1-Cre and A2a-Cre intact mice. Whole-cell patch clamp recordings were made in ex vivo slices from SPNs transduced with hM3Dq or EGFP. (J) Bath application of CNO $(10 \mu \mathrm{M})$ gradually increased the number of APs induced by brief somatic current pulses in both dSPNs and iSPNs transduced with hM3Dq, but no increase was observed in the control group. RM 2-way ANOVA ( $n=7$ cells per data set): effect of group, $F_{(2,18)}=15.80$, $P=0.0001$; time (effect of CNO), $F_{(4,72)}=11.29, P=0.0001$; interaction, $F_{(8,72)}=3.446, P=0.0021$. ${ }^{*} P<0.05 ;{ }^{* *} P<0.01$ for the effect of CNO in dSPN-hM3Dq vs. dSPN-EGFP; \#\#\# $P$ < 0.001 for the effect of CNO in iSPN-hM3Dq vs. dSPN-GFP. (K) Representative traces of AP responses to current injection, at baseline and after CNO application, in hM3Dq-transduced dSPNs and iSPNs and an EGFP-transduced dSPN.

both therapeutic-like and dyskinetic responses to L-DOPA. Furthermore, our results challenge the notion that dSPN overactivity alone can explain LID, showing that full-blown dyskinesias only are achieved by interventions mimicking the action of L-DOPA on both pathways.

\section{Results}

Validation of the chemogenetic approach in intact animals. To chemogenetically control the activity of direct and indirect pathways in vivo, we used BAC transgenic mice expressing Cre recombinase under the control of SPN type-specific promoters (18). Creinducible adeno-associated viral (AAV) vectors coding for the activatory Gq-coupled human M3 muscarinic receptor (hM3Dq), a type of Designer Receptors Exclusively Activated by Designer Drugs (DREADDs; herein referred to as Gq-DREADD) (19), were injected unilaterally into the dorsolateral striatum. In a first experiment, the viral vector was delivered to intact A2a-Cre and D1-Cre mice. To validate the functionality of the Gq-DREADD vector, we used behavioral, histomolecular, and electrophysiological assays. Four weeks after AAV-DIO-hM3Dq-mCherry transduction, mice were perfusion-fixed 30 minutes after vehicle or clozapine $\mathrm{N}$-oxide (CNO) treatment $(1 \mathrm{mg} / \mathrm{kg}$, i.p.) for immunohistochemical studies. In the AAV-transduced SPNs (identified by the fluorescent tag mCherry, cf. Figure 1, A, D, and G), treatment with $\mathrm{CNO}$ induced expression of the phosphorylated (active) form of ERK1/2 (p-ERK), which is a downstream target of this type of DREADD $(19,20)$ (Figure 1, D-F). Positive immunostaining for p-ERK did not occur in vehicle-treated mice (Figure $1, \mathrm{G}-\mathrm{I}$ ), ruling out a constitutive activity of the DREADD construct (21). Electrophysiological recordings were performed ex vivo using slices from D1-Cre and A2a-Cre mice transduced with hM3Dq or a control vector (AAV-DIO-EGFP) (Figure 1, $\mathrm{J}$ and 
iSPN-hM3Dq, motor activity
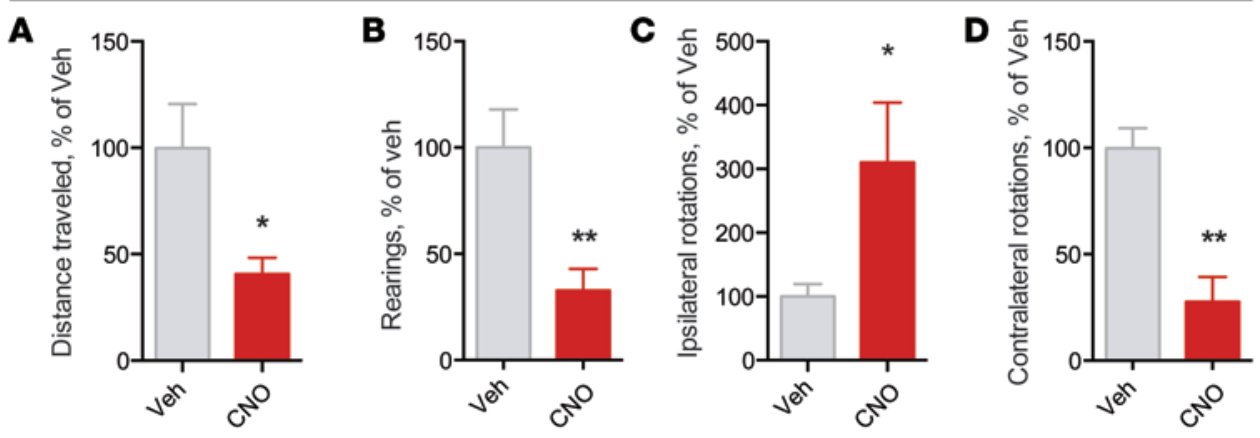

iSPN-EGFP, motor activity
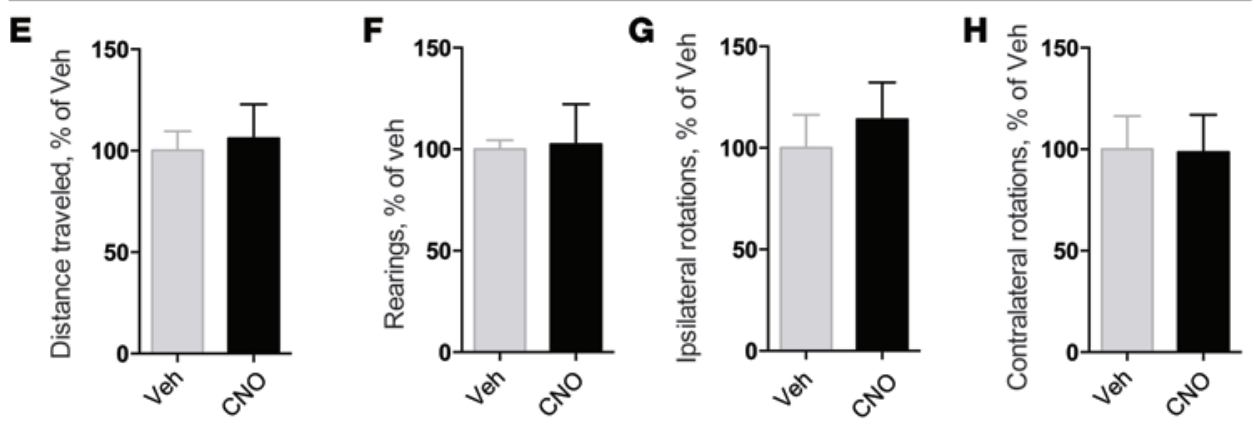

dSPN-hM3Dq, motor activity
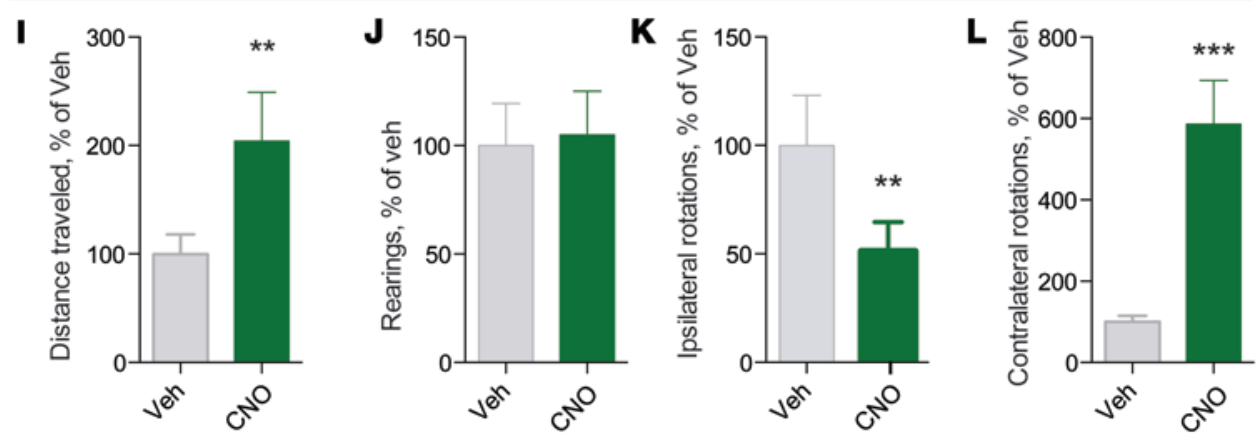

dSPN-EGFP, motor activity
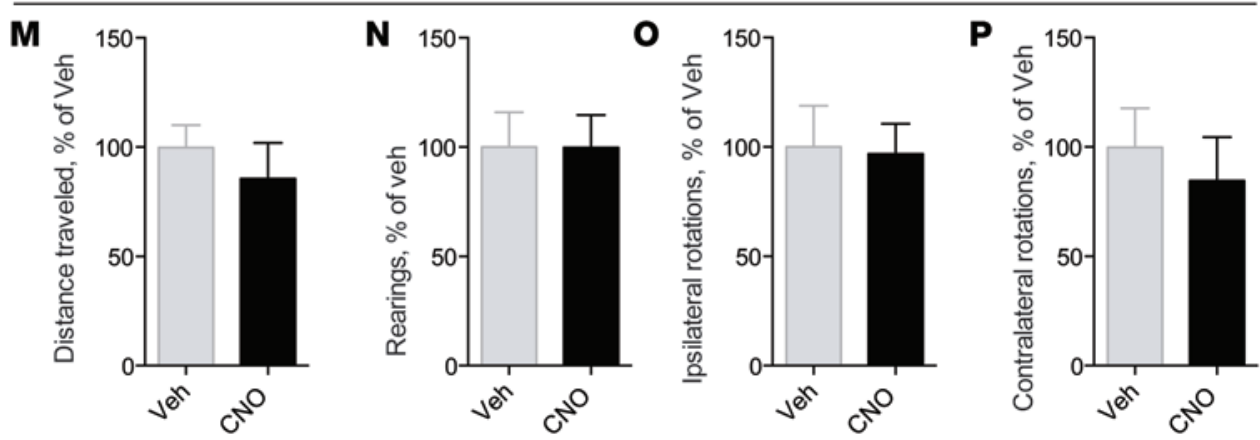

Figure 2. Motor activity in an open field upon Gq-DREADD-mediated activation of iSPNs or dSPNs in intact mice. Results from AAV-transduced A2a-Cre and D1-Cre mice are presented in $\mathbf{A}-\mathbf{H}$ and $\mathbf{I}-\mathbf{P}$, respectively. (A-D) iSPN-hM3Dq stimulation with CNO causes reductions in distance travelled (A) and rearing events (B) along with an ipsilateral rotational bias (C and D). Veh, vehicle. (E-H) Treatment with CNO is without effect when iSPNs are transduced with a control vector (EGFP). (I-L) dSPNhM3Dq stimulation with CNO causes an increase in distance travelled (I), no significant change in vertical activity (J), and a contralateral rotational bias (K and $\mathbf{L}$ ). (M-P) Treatment with CNO does not affect any motor behavior when dSPNs are transduced with the control vector (EGFP). Data recorded after treatment with CNO $(1 \mathrm{mg} /$ $\mathrm{kg}$ ) are expressed as a percentage of those measured after vehicle administration in the same mice. Values show mean + SEM from the following numbers of mice: $n=7$ (iSPN-hM3Dq); $n=6$ (iSPN-EGFP); $n=11$ (dSPNhM3Dq); $n=5$ (dSPN-EGFP). Paired 2-tailed Student's $t$ test: ${ }^{*} P<0.05$ ${ }^{* *} P<0.01 ;{ }^{* * *} P<0.001$ for $C N O$ vs. vehicle. NS indicates $P>0.05$.
$\mathrm{K})$. Bath application of CNO $(10 \mu \mathrm{M})$ gradually increased the frequency of action potentials (APs) induced by brief somatic current pulses in both dSPNs and iSPNs transduced with hM3Dq. In contrast, no increase was observed in SPNs transduced with the control vector (Figure 1, J and $\mathrm{K}$ ).
At the behavioral level, Gq-DREADD-mediated activation of indirect and direct pathway neurons was found to exert opposite motor effects in an open-field test. Thus, hM3Dq-mediated stimulation of iSPNs by $\mathrm{CNO}$ reduced both the distance travelled (Figure $2 \mathrm{~A}$ ) and the number of rearing events (Figure 2B). Moreover, CNO 
treatment induced a rotational bias toward the side ipsilateral to the DREADD transduction (Figure 2C) paralleled by a reduction in contralateral rotations (Figure 2D). Mice injected with a control vector did not respond to $\mathrm{CNO}$ treatment (Figure 2, E-H). In intact D1-Cre mice, Gq-mediated activation of dSPNs increased the distance travelled (Figure 2I), though not the number of rearings (Figure 2J). In the same animals, treatment with CNO induced a turning bias to the direction contralateral to the DREADD transduction. Thus, the number of ipsilateral rotations decreased (Figure $2 \mathrm{~K}$ ), while contralateral rotations increased (Figure 2L) after the administration of CNO. No differences between $\mathrm{CNO}$ and vehicle treatment were found on any motor parameter in mice injected with the control vector (Figure 2, M-P). Taken together, these data prove the validity of our chemogenetic approach to affecting both striatal signaling and motor behavior in mice. The results from the open-field test show that Gq-DREADD-mediated activation of dSPNs and iSPNs oppositely modulates whole-body movements, in keeping with effects reported following optogenetic stimulation of these neuronal systems in mice (8).

Gq-DREADD mediated activation of iSPNs and dSPNs in a mouse model of PD. We next applied the same chemogenetic approach to a unilateral mouse model of parkinsonism that is widely used for studies of striatal signaling and plasticity (22-29). BAC-transgenic mice sustained 6-hydroxydopamine (6-OHDA) lesions of the nigrostriatal DA pathway on one side of the brain, and AAV-DIO-hM3Dq was injected into the DA-denervated striatum 3 weeks later. We first examined the effects of chemogenetic SPN stimulation on whole-body movements in the open-field test. As seen in intact mice, activation of iSPNs by CNO significantly decreased both horizontal and vertical activity (Figure 3, A and B) and enhanced ipsilateral rotation (Figure $3 \mathrm{C}$ ) while reducing contralateral rotational behavior (Figure 3D). Opposite effects were produced by hM3Dq-mediated activation of dSPNs. In this case, treatment with CNO markedly increased both horizontal and vertical activity (Figure 3E, distance travelled; Figure 3F, rearing events), reduced the number of ipsilateral turns (Figure 3G), and caused a dramatic increase in contralateral rotations (Figure $3 \mathrm{H}$ ). We noticed that the chemogenetic stimulation of dSPNs yielded larger motor changes in parkinsonian mice compared with intact animals (cf. measures of vertical activity and contralateral rotations in Figures 2 and 3). In contrast, motor changes induced by iSPN stimulation did not seem to be affected by the presence of a nigrostriatal DA lesion. We concluded that denervation-dependent hyperactivity of iSPNs (30) did not occlude the stimulatory effects of Gq-DREADD in PD mice.

We next asked whether the therapeutic effect of L-DOPA could be mimicked or modulated by SPN type-specific chemogenetic stimulation. In hemiparkinsonian mice, hypokinesia manifests as a reduced use of the limbs contralateral to the lesion in all types of spontaneous movement, and therapeutic doses of L-DOPA promptly ameliorate this feature (31). To quantify lesioninduced hypokinesia, we used a test of spontaneous forelimb use during vertical exploration (the cylinder test; ref. 32). As shown in Figure 4, 6-OHDA-lesioned mice of both genotypes exhibited a similar impairment in contralateral forelimb use, which was similarly improved by L-DOPA ( $3 \mathrm{mg} / \mathrm{kg})$. Activation of iSPNs by $\mathrm{CNO}$ did not worsen the lesion-induced limb hypokinesia (Fig- ure 4A, nonsignificant difference for CNO vs. vehicle). However, CNO prevented the beneficial effect of L-DOPA on contralateral forelimb use (Figure 4A, nonsignificant difference for $\mathrm{CNO}+\mathrm{L}-$ DOPA vs. vehicle). In 6-OHDA-lesioned D1-Cre mice, activation of dSPNs by CNO restored forelimb use symmetry, fully mimicking the therapeutic effect of L-DOPA (Figure 4B, $P<0.001$ for $\mathrm{CNO}$ vs. vehicle, and nonsignificant difference between $\mathrm{CNO}$ and L-DOPA). Combined treatment with CNO and L-DOPA biased animals toward a preferential use of the contralateral forelimb (Figure 4B, $P<0.01$ for CNO+L-DOPA vs. CNO).

Modulation of LID by pathway-specific chemogenetic stimulation. To determine whether pathway-specific stimulation can modulate LID, 6-OHDA-lesioned mice of both genotypes were injected with the hM3Dq vector and then treated with ascending doses of L-DOPA (1, 3 and $12 \mathrm{mg} / \mathrm{kg}$, 1 week of daily treatment with each dose). The dyskinesia dose response was very similar between genotypes. Thus, treatment with the lowest dose of L-DOPA ( $1 \mathrm{mg} / \mathrm{kg}$ ) only induced incipient abnormal involuntary movements (AIMs) in both A2a-Cre and D1-Cre mice (cf. Veh+LD data in Figure 5, A and B, respectively). CNO administration completely suppressed these AIMs in A2a-Cre mice (Figure 5A, $P<0.05$ ), but markedly enhanced them in D1-Cre mice (Figure 5B, $P<0.01$ ). The dyskinesia-enhancing effect of dSPN stimulation was clear-cut on all types of involuntary movements, i.e., axial, limb, and orofacial AIMs (see color-coded specification of AIM subtypes in Figure 5, bar diagram). The dose of $3 \mathrm{mg} / \mathrm{kg}$ L-DOPA is typically used to induce LID in this animal model (25, 29). As expected, this L-DOPA dosage elicited moderate to severe dyskinesia in both genotypes (cf. Veh+LD data in Figure 5, C and D). Also, these overt dyskinesias were bidirectionally modulated by pathway-specific stimulation. Thus, combined treatment with CNO and L-DOPA suppressed and enhanced the AIM scores in A2a-Cre and D1-Cre mice, respectively (Figure 5, C and D; P < 0.05 for the effect of $\mathrm{CNO}$ in both genotypes). During the last week of treatment, LID severity was pushed to a maximum using $12 \mathrm{mg} / \mathrm{kg}$ L-DOPA. With this dosage, all animals reached the highest severity score on at least one AIM subtype at the peak of the dyskinesia-time curve (cf. scores at 40-60 minutes in Figure 5, E and F). In A2a-Cre mice, cotreatment with CNO and L-DOPA caused a sustained reduction in AIM scores (Figure 5E; $P<$ 0.001), significantly affecting all dyskinesia subtypes (Figure 5E, bar diagram). In D1-Cre mice, the administration of CNO did not further exacerbate the peak severity of LID (cf. scores at 40-60 minutes in Figure 5F), but it resulted in higher AIM scores at 100-180 minutes, hence in larger AIM scores per session (Figure $5 \mathrm{~F}$, bar diagram, $P<0.01$ ). These data show, for what we believe is the first time, that dyskinetic behaviors induced by L-DOPA in a rodent model of PD can be oppositely modulated by activating direct or indirect pathway SPNs through Gq-DREADD.

Induction of dyskinesia through dSPN activation depends on the signaling pathway recruited by the DREADD. We next asked whether dSPN activation would be sufficient to elicit dyskinesia in the absence of L-DOPA, as implied by recent hypotheses (33-35). To this end, D1-Cre mice sustained 6-OHDA lesions followed by striatal delivery of either hM3Dq or an activatory DREADD that couples to Gs-like (Gs-DREADD), the modified rat M3 muscarinic receptor (rM3Ds) (36). Mice were treated chronically with CNO $(1,2$, and 5 
iSPN-hM3Dq, motor activity
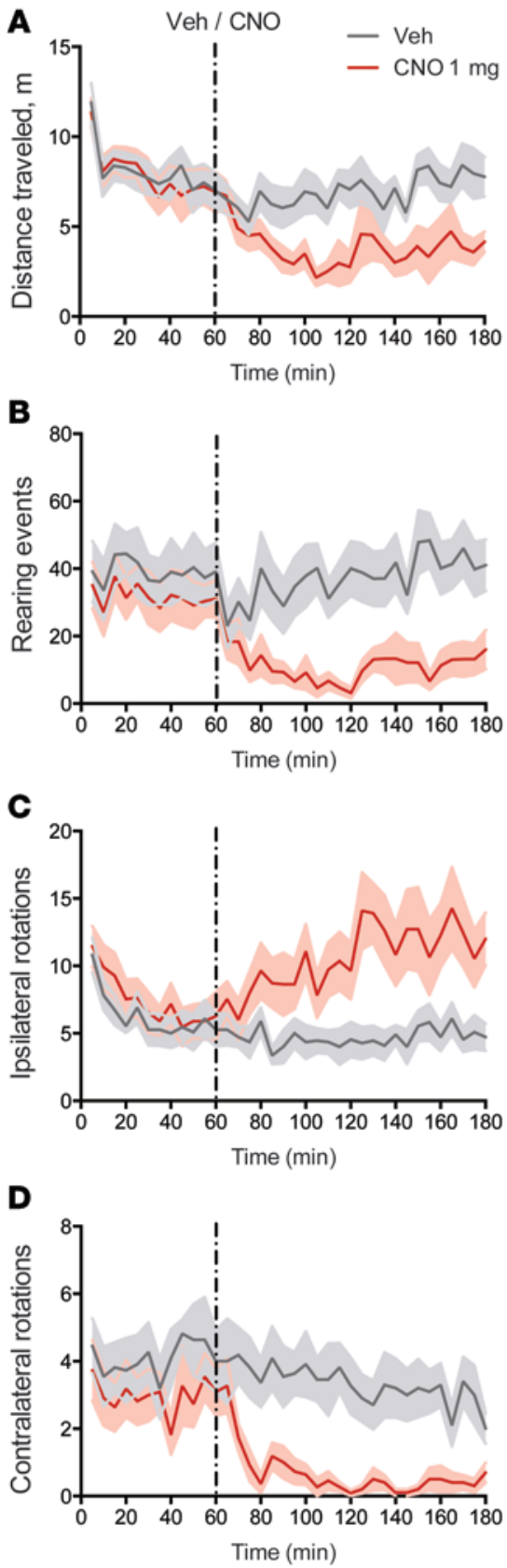
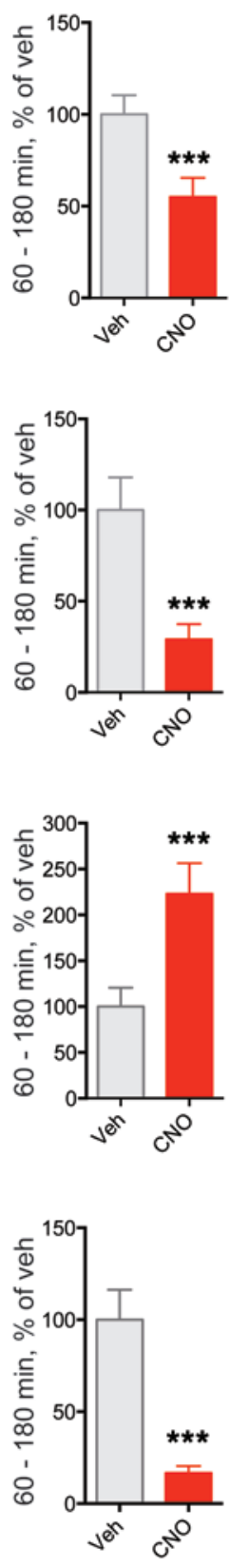

dSPN-hM3Dq, motor activity
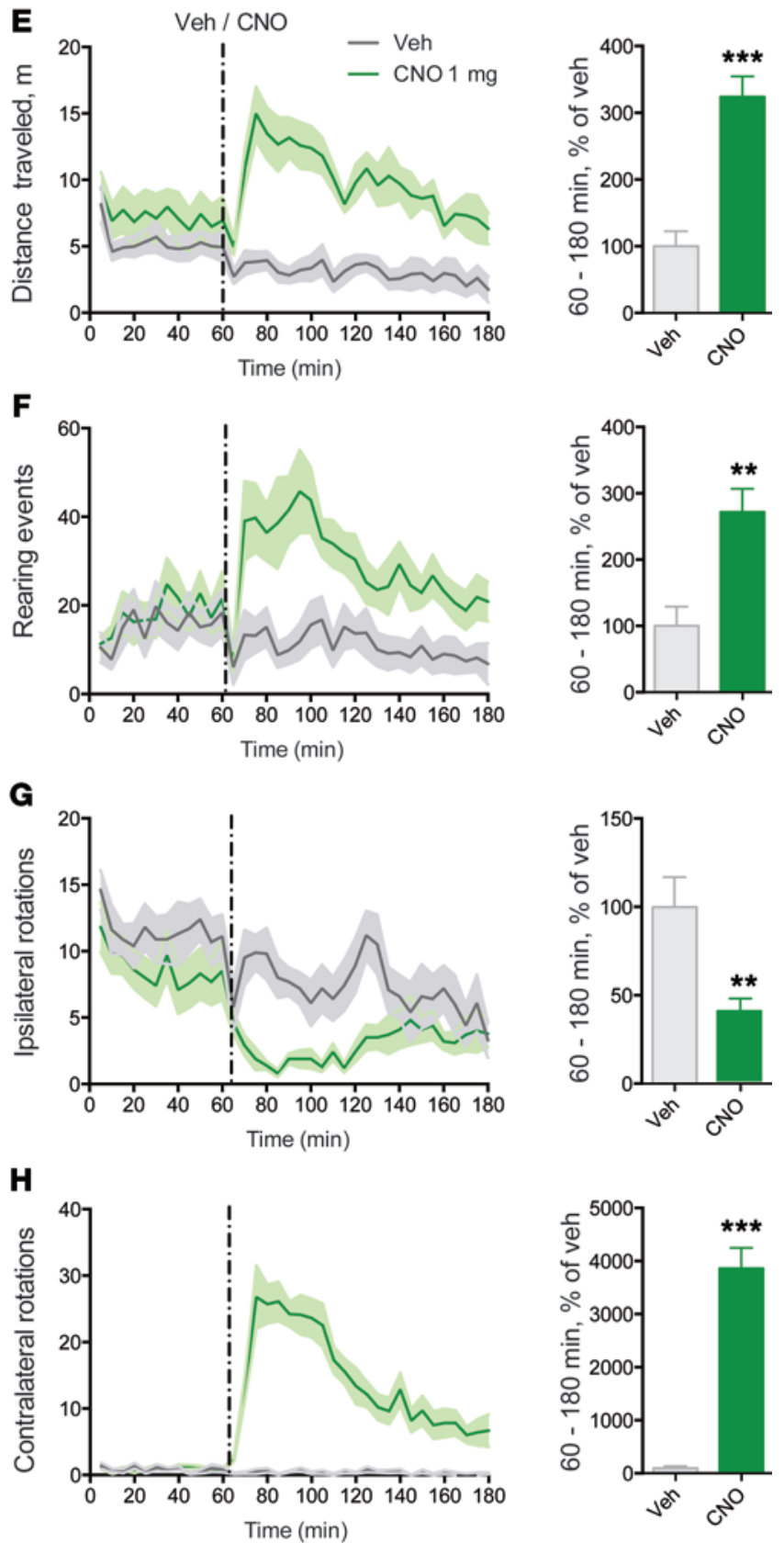

Figure 3. Motor activity in an open field upon Gq-DREADD-mediated activation iSPNs or dSPNs in 6-OHDA-lesioned mice. Data were acquired from A2a-Cre (A-D) or D1-Cre mice (E-H) transduced with the hM3Dq vector. Line diagrams show the time course of behavioral recordings, and hatched lines indicate the time point when mice were injected with either CNO or vehicle. Bar diagrams show recordings after treatment with CNO (1 mg/kg) expressed as a percentage of vehicle values. RM 2-way ANOVA: $(\mathbf{A}, \mathbf{E})$ distance travelled: $(\mathbf{A})$ iSPN: treatment, $F_{(1,10)}=21.08, P<0.01 ;$ time, $F_{(35,350)}=7.930, P<0.001$; interaction, $F_{(35,350)}=4.134, P<0.001$. (E) dSPN: treatment, $F_{(1,9)}=75.41, P<0.001$; time, $F_{(35,315)}=3.319, P<0.001$; interaction, $F_{(35,315)}=5.792, P<0.001$. (B, F) Rearing: (B) iSPN: treatment, $F_{(1,10)}=32.66, P<0.001$; time, $F_{(35,350)}=3.633, P<0.001$; interaction, $F_{(35.350)}=3.809, P<0.001$. (F) dSPN: treatment, $F_{(1,9)}=16.94, P<0.01$; time, $F_{(35,315)}=3.817, P<0.001$; interaction, $F_{(35,315)}=3.860, P<0.001$. (C, G) Ipsilateral rotations: $(\mathbf{C})$ iSPN: treatment, $F_{(1,10)}=25.04$, $P<0.001$; time, $F_{(35,350)}=2.463, P<0.001$; interaction, $F_{(35,350)}=3.432, P<0.001$. (G) dSPN: treatment, $F_{(1,9)}=11.97, P<0.01 ;$ time, $F_{(35,315)}=8.081, P<0.001$; interaction, $F_{(35,315)}=1.868, P<0.01$. (D, H) Contralateral rotations: $(\mathbf{D})$ iSPN: treatment, $F_{(1,10)}=21.69, P<0.001$; time, $F_{(35,350)}=3.668, P<0.001$; interaction $F_{(35,350)}=2.442, P<0.001$. (H) dSPN: treatment, $F_{(1,9)}=96.77, P<0.001$; time, $F_{(35,315)}=23.40, P<0.001$; interaction, $F_{(35,315)}=24.81, P<0.001$. Bar diagrams represent recordings made from 60 to 180 minutes. Data are expressed as mean + SEM from A2a-Cre mice $(n=11)$ and $D 1-C$ re mice $(n=10)$. Paired 2 -tailed Student's $t$ test: ${ }^{* *} P<0.01 ;{ }^{* *} P<0.001$ for CNO vs. vehicle. 
A

iSPN-hM3Dq

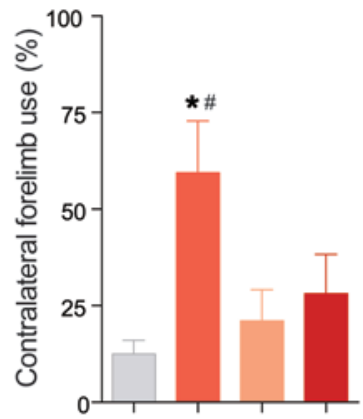

$\mathrm{CNO}(1 \mathrm{mg} / \mathrm{kg})-\quad-++$
B

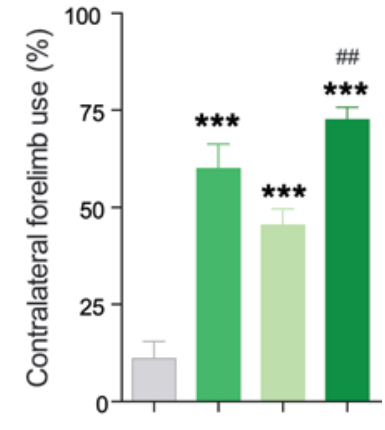

$\mathrm{CNO}(1 \mathrm{mg} / \mathrm{kg})-\quad-\quad++$

$\mathrm{LD}(3 \mathrm{mg} / \mathrm{kg})-+\quad+$ $\mathrm{mg} / \mathrm{kg}$, 4 days of treatment with each dose) and examined on the AIM rating scale (Figure 6). In hM3Dq-transduced mice, treatment with $\mathrm{CNO}$ induced a dose-dependent development of mild AIM scores, whose severity and topographic distribution did not, however, approximate those typical for L-DOPA treatment (cf. grayshaded area in Figure 6A, representing the mean \pm SEM interval of AIM scores induced by $3 \mathrm{mg} / \mathrm{kg}$ L-DOPA in D1-Cre-lesioned mice). When axial, limb, and orofacial AIMs were analyzed separately, it became apparent that the dyskinesia score accumulated in each session was entirely dependent on the orofacial subtype, while axial and limb AIMs were very low or totally absent upon CNO stimulation of hM3Dq-dSPNs (cf. total AIM scores vs. AIM subtypes in Figure 6A) (see Supplemental Videos 1-3; supplemental material available online with this article; doi:10.1172/JCI90132DS1).

Stimulation of dSPNs via rM3Ds resulted in a dose-dependent induction of axial, limb, and orolingual AIMs (Figure 6B, and Supplemental Video 4). With the highest dose of CNO $(5 \mathrm{mg} / \mathrm{kg})$, the AIM scores accrued in 1 test session were in the same range as those induced by $3 \mathrm{mg} / \mathrm{kg} \mathrm{L}$-DOPA (cf. gray-shaded areas in Figure 6B and Supplemental Video 5). This pertained to both the total AIM score and the individual AIM subtypes (Figure 6B). However, the time course of CNO-induced AIMs was more prolonged than LID, which typically subsides by 120 minutes after drug administration (cf. AIMs time curve upon treatment with 3 $\mathrm{mg} / \mathrm{kg}$ L-DOPA in Figure 5D). Indeed, in the Gs-DREADD group, AIMs induced by $5 \mathrm{mg} / \mathrm{kg}$ CNO reached their maximum severity 60 minutes after drug injection and then remained stable until the end of the testing session (Figure 6D). We therefore examined peak AIM scores in the different groups and found them to be markedly lower upon rM3Ds-dSPN stimulation compared with L-DOPA treatment $(3 \mathrm{mg} / \mathrm{kg}$ ) (total AIM score at $60 \mathrm{~min}$ utes, 2.5 vs. 6.5 , respectively, cf. Figure $6 \mathrm{D}$ vs. Figure 5D). Comparisons of CNO-induced AIMs in the hM3Dq and rM3Ds groups are presented in Figure 6, C and D, where the effects of $1 \mathrm{mg}$ / $\mathrm{kg}$ and $5 \mathrm{mg} / \mathrm{kg} \mathrm{CNO}$ are depicted. With both doses of CNO, the total AIM score per session was significantly larger when dSPNs were stimulated via Gs-DREADD compared with Gq-DREADD. As particularly evident from Figure 6D (bar diagram), the difference between hM3Dq- and rM3Ds-mediated dyskinesias was accounted for by axial and limb AIMs, whereas orofacial AIMs had about the same severity with both constructs.
Figure 4. Gq-DREADD-mediated activation of iSPNs or dSPNs oppositely modulates therapeutic-like effects of L-DOPA in 6-OHDAlesioned mice. The beneficial effect of L-DOPA is represented by an increased use of the parkinsonian forelimb (percentage of contralateral forelimb use) in the cylinder test. Data were acquired 20 to 25 minutes after the administration of L-DOPA (LD) (3 mg/ $\mathrm{kg})$, CNO ( $1 \mathrm{mg} / \mathrm{kg})$, or vehicle, as given alone or in combination. (A) Contralateral forelimb use in 6-OHDA-lesioned and hM3Dq-transduced A2a-Cre mice $(n=7)$. RM 1-way ANOVA: treatment, $F_{(3,18)}=7.219, P<0.01$. Post hoc Tukey's test: ${ }^{*} P<0.05$ vs. veh+veh; ${ }^{*} P<0.05$ vs. CNO+veh. (B) Contralateral forelimb use in 6-OHDA-lesioned and hM3Dq-transduced D1-Cre mice $(n=11)$. RM 1-way ANOVA: treatment, $F_{(3,30)}=31.24, P<0.001$. Post hoc Tukey's test: ${ }^{* * *} P<0.001$ vs. veh+veh; $\# P<0.01$ vs. CNO+veh.

We next asked whether combining rM3Ds-dSPN stimulation with pharmacological stimulation of $\mathrm{D} 2$ receptors (thus mimicking the action of L-DOPA on both dSPNs and iSPNs) would replicate the full severity of LID. To this end, D1-Cre 6-OHDA-lesioned animals transduced with the rM3Ds vector were injected with CNO $(1 \mathrm{mg} / \mathrm{kg})$, the D2 agonist quinpirole at a postsynaptically active dose $(0.5 \mathrm{mg} / \mathrm{kg})$, or both treatments in a randomized order. The plot of AIM scores per monitoring period revealed marked differences between treatments (Figure 7A). Both quinpirole and CNO induced moderate AIM scores, although peaking at distinct time points (40 minutes and 100 minutes, respectively; Figure 7A). Combined treatment with quinpirole and CNO elicited conspicuous and sustained dyskinesias, reaching maximum severity by 40 minutes and remaining quite severe until the end of the test session (Figure 7A, $P<0.05$ for combined treatment vs. CNO or quinpirole on each monitoring period) (Supplemental Video 6). A highly significant difference between the combined quinpirole+CNO treatment and each drug given alone was found on both the total AIM scores per session (Figure 7B) and each individual AIM subtype (see gray-coded representation of axial, limb, and orofacial AIMs in Figure 7B). Upon quinpirole+CNO treatment, the sum of AIM scores per session greatly exceeded the values produced by the highest dose of L-DOPA $(12 \mathrm{mg} / \mathrm{kg} \mathrm{L-DOPA}$, see gray bar in Figure $7 \mathrm{~B})$ and the AIM score per monitoring period reached the same peak value ( 10, see dotted line in Figure $7 \mathrm{~A})$.

Finally, we verified whether a chemogenetic stimulation of dSPNs could also induce dyskinesia in intact mice unilaterally transduced with one or the other DREADD (Supplemental Figure 1). The administration of CNO to intact animals induced only very mild or no AIMs. No significant differences were observed between groups transduced with hM3Dq or rM3Ds (see Supplemental Figure 1, A and B).

Signaling pathways activated by the stimulation of $h M 3 D q$ and $r M 3 D$ s. The data above suggest that the dyskinetic outcome of dSPN stimulation in PD models depends on the signaling pathway that is recruited by the DREADD construct. To explore this hypothesis, we compared molecular signaling events induced by the 2 activatory DREADDs in 6-OHDA-lesioned mice (Figure 8). To this end, we counted p-ERK-immunoreactive neurons within transduced striatal areas (depicted in Figure 8D). In the same areas, we counted the number of neurons immunoreactive for the 

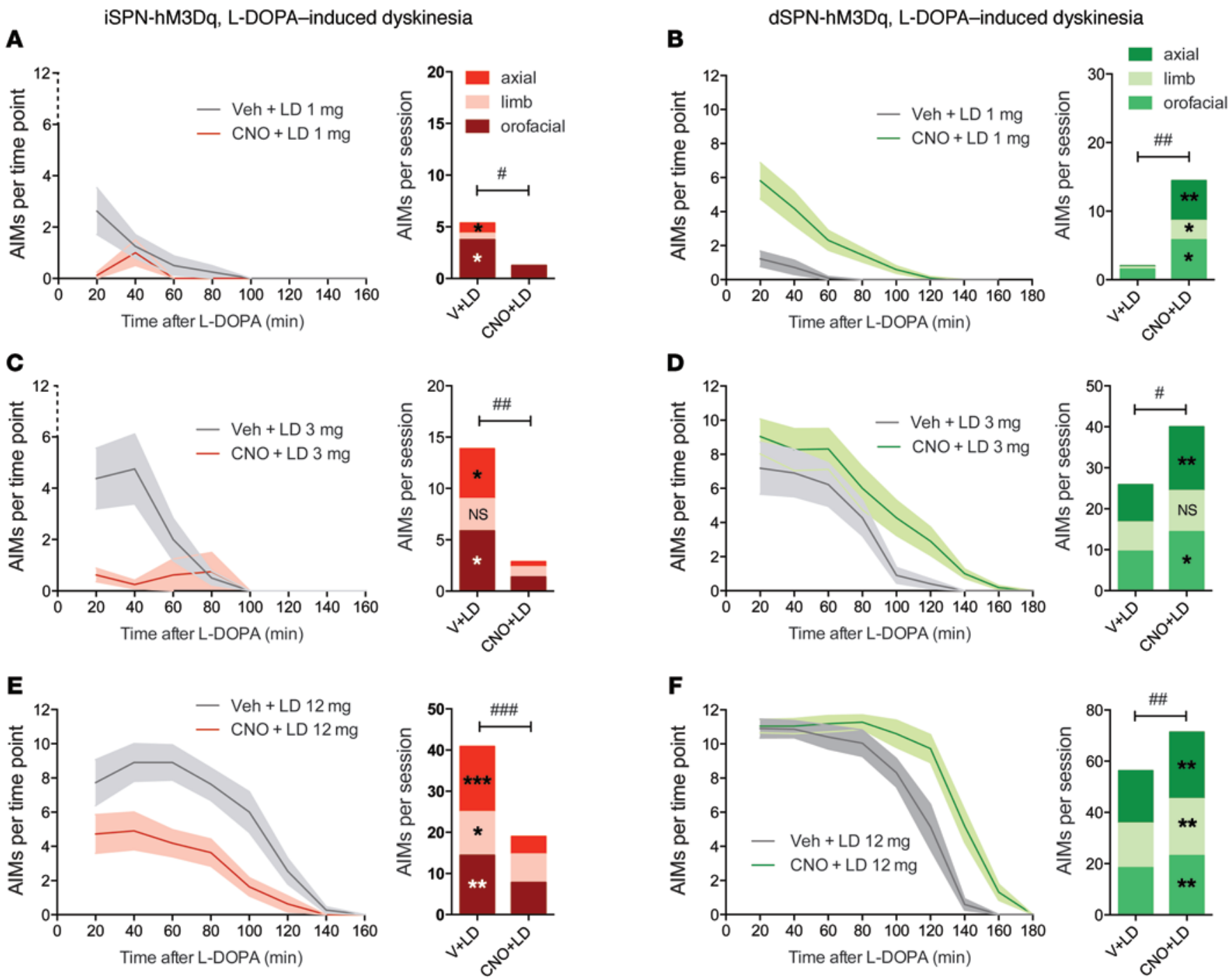

Figure 5. Modulation of LID behaviors in 6-OHDA-lesioned mice by Gq-DREADD-mediated activation of iSPNs or dSPNs. AIMs of increasing severity were induced with escalating doses of L-DOPA, i.e., $1 \mathrm{mg} / \mathrm{kg}$ ( $\mathbf{A}$ and $\mathbf{B}), 3 \mathrm{mg} / \mathrm{kg}$ (C and $\mathbf{D})$, and $12 \mathrm{mg} / \mathrm{kg}$ (E and $\mathbf{F}$ ). Data from 6-OHDA-lesioned and hM3Dq-transduced A2a-Cre mice (red line/bars) and D1-Cre mice (green line/bars) are presented in the left-hand column and right-hand column, respectively. Time courses of axial, limb, and orofacial AIM scores during the 180-minute test sessions are shown in A, C, and E (A2a-Cre mice) and B, D, and $\mathbf{F}$ (D1-Cre mice). RM 2-way ANOVA ( $n=8$-11 per group): (A and B) L-DOPA (1 mg/kg): (A) iSPN: treatment, $F_{(1,7)}=5.914, P<0.05$; time, $F_{(7,49)}=7.913, P<0.001$; interaction, $F_{(7,49)}=4.564, P<0.001$. (B) dSPN: treatment, $F_{(1,10)}=14.67, P<0.01$; time, $F_{(7.70)}=28.60, P<0.001$; interaction, $F_{(8,80)}=9.632, P<0.001$. (C and D) L-DOPA (3 mg/kg): (C) iSPN: treatment, $F_{(1,7)}=14.07, P<0.01$; time, $F_{(7,49)}=8.015, P<0.001$; interaction, $F_{(7,49)}=7.406, P<0.001$. (D) dSPN: treatment, $F_{(1,10)}=7.442, P<0.05$; time, $F_{(8,80)}=38.45, P<0.001$; interaction, $F_{(8,80)}=2.038, P=0.052$. (E and $\left.\mathbf{F}\right)$ L-DOPA (12 mg $\left./ \mathrm{kg}\right)$ : (E) iSPN: treatment, $F_{(1,10)}=34.32$, $P<0.001$; time, $F_{(7,70)}=28.66, P<0.001$; interaction, $F_{(7,70)}=7.682, P<0.001$. (F) dSPN: treatment, $F_{(1,10)}=14.03, P<0.01 ;$ time, $F_{(8,80)}=155.9, P<0.001$; interaction, $F_{(8,80)}=7.190, P<0.001$. Bar diagrams represent the sum of AIM scores per session, representing axial, limb and orofacial components using different colors. Paired 2-tailed Student's $t$ test: ${ }^{\# P}<0.05$; ${ }^{\#} P<0.01 ; \# \# P<0.001$ for CNO+LD vs. veh+LD on the total AIM score per session. ${ }^{*} P<0.05$; ${ }^{* *} P<0.01$; ${ }^{* *} P<0.001$ for CNO+LD vs. veh+LD on each individual AIM subtype.

phosphorylated form of the PKA substrate consensus sequence (p-PKA substrate), which has been validated as a marker of cAMP/ PKA signaling activation (37). Analyses were carried out on striata harvested at 30 minutes or 140 minutes after $\mathrm{CNO}$ administration, representing time points when AIMs were present in both hM3Dq and rM3Ds groups (30 minutes) or only in the latter (140 minutes). Thirty minutes after $\mathrm{CNO}$ administration, the number of p-ERK-positive neurons was 3-fold larger in striata transduced with Gs- compared with Gq-DREADD constructs (Figure 8, A and C). The difference between types of DREADD was even clearer at 140 minutes after $\mathrm{CNO}$ injection, at which time point the activation of p-ERK had subsided in the hM3Dq group, but was still significant in the rM3Ds group (Figure 8, A and C). The expression of p-PKA substrate was quite different between chemogenetic stimuli. Thus, dSPN stimulation via Gq-DREADD did not induce any expression of p-PKA substrate immunoreactivity above background levels (Figure 8, B and E). In contrast, Gs-DREADD stimulation caused a significant activation of p-PKA substrates at 30 minutes and sparse immunoreactivity for this marker at $140 \mathrm{~min}$ utes after CNO injection (Figure 8, B and E). 
A
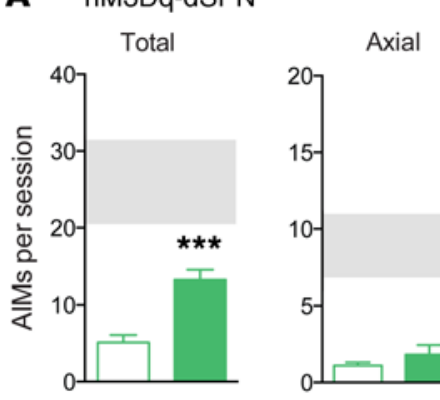

Axial

Limb

Orofacial

CNO $1 \mathrm{mg}$

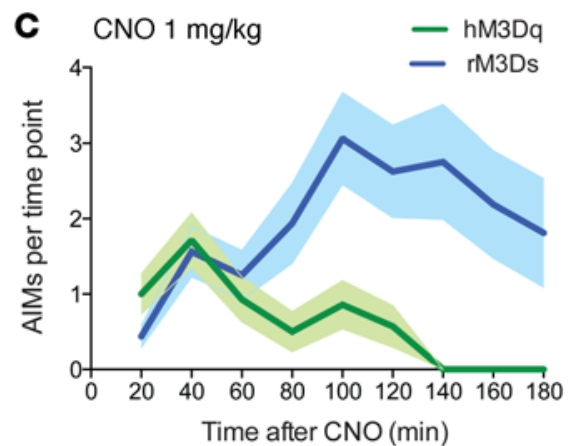

B rM3Ds-dSPN

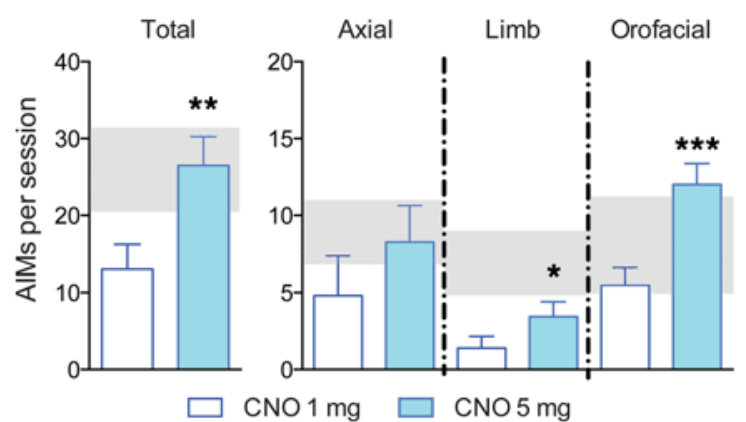

D $\mathrm{CNO} 5 \mathrm{mg} / \mathrm{kg}$

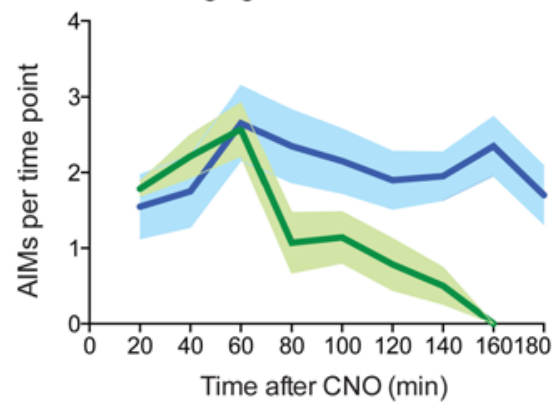

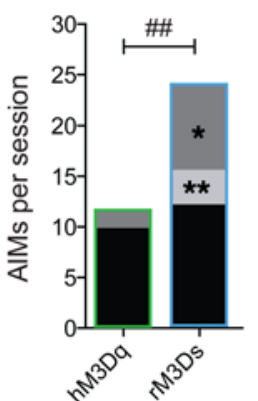

Figure 6. Induction of dyskinetic behaviors by Gq- versus Gs-DREADD-dependent activation of dSPNs in L-DOPA-naive 6-OHDA-lesioned mice. (A and B) Bar diagrams show the total AIM score per session and the separate axial, limb, and orofacial scores following treatment with the lowest and highest doses of CNO tested in this experiment ( 1 and $5 \mathrm{mg} / \mathrm{kg}$, respectively; see horizontal captions). Gray-shaded areas represent the range (mean \pm SEM) of AIM scores induced by a therapeutic dose of L-DOPA (3 mg/kg) in D1-Cre 6-OHDA-lesioned mice (cf. Figure 5D). Data are shown as mean + SEM from $n=7$ mice transduced with hM3Dq and $n=8$ mice transduced with rM3Ds. Paired 2-tailed Student's $t$ test: ${ }^{*} P<0.05 ;{ }^{* *} P<0.01$; ${ }^{* * *} P<0.001$ for CNO $5 \mathrm{mg} / \mathrm{kg}$ vs. CNO $1 \mathrm{mg} / \mathrm{kg}$. (C and D) Direct comparisons of CNO-induced AIMs in mice transduced with the 2 different types of DREADD. (C and D) Time course of AIMs after CNO injection (scored every 20 minutes until 180 minutes). Two-way RM ANOVA: (C) CNO (1 mg/kg): DREADD type, $F_{(1,13)}=$ 6.737, $P<0.05$; time, $F_{(8,104)}=3.557, P<0.01$; interaction, $F_{(8.104)}=3.557, P<0.001$. (D) CNO (5 mg/kg): DREADD type $F_{(1.15)}=4.352, P=0.054 ;$ time, $F_{(8,120)}=7.903, P<0.001$; interaction, $F_{(8,120)}=6.549, P<0.001$. Bar diagrams show the sum of AIM scores per session, and scores accrued on each of axial, limb, and orofacial AIMs are represented using different shades of gray. Unpaired 2-tailed Student's $t$ test: total AIMs per session: ${ }^{P}<<0.05$; ${ }^{\# \# P}<0.01$ vs. hM3Dq. Individual AIM subtypes: ${ }^{*} P<0.05 ;{ }^{* *} P<0.01$ vs. hM3Dq.

Electrophysiological effects of DREADD-induced dSPN activation. To shed further light on the functional impact of rM3Ds versus hM3Dq stimulation, we performed electrophysiological recordings from dSPNs in corticostriatal slices. Transduced dSPNs were identified by somatic expression of mCherry and EGFP (see Methods). The basic electrophysiological properties of recorded neurons conformed to values reported from dSPNs in previous studies $(38,39)$ and did not differ between cells transduced with either DREADD construct (Supplemental Table 1). In dSPNs transduced with either hM3Dq or rM3Ds, bath application of CNO $(10 \mu \mathrm{M})$ did not evoke any AP per se, but it gradually increased the generation of APs in response to brief current pulses (Figure 9, $\mathrm{A}$ and $\mathrm{B})$. In both intact and 6-OHDA-lesioned mice transduced with either hM3Dq or rM3Ds, the average frequency of induced APs increased markedly between baseline and 20-25 minutes after CNO application (CNO, Figure 9, C and D). In groups transduced with the Gq-DREADD, the effect of CNO on evoked APs was identical between intact and 6-OHDA-lesioned animals (Figure 9, A, C, and E). In contrast, in Gs-DREADD-transduced groups, the effect of $\mathrm{CNO}$ was significantly larger in the presence of a DA-denervating lesion (Figure 9, B, D, and F). Taken together, these data indicate that chemogenetic stimulation of dSPNs via
Gq-DREADD or Gs-DREADD enhances the response to depolarizing stimuli and that the effect of Gs-DREADD stimulation is significantly potentiated in the presence of a DA-denervating lesion.

\section{Discussion}

Parkinsonism and dyskinesia have been attributed to opposite imbalances in the activity of dSPNs versus iSPNs. Thus, hypo/ bradykinesia would result from underactivity of dSPN and overactivity of iSPN, as caused by deficient stimulation of D1 and D2 receptors, respectively $(6,8)$. During the first years of treatment, L-DOPA pharmacotherapy is apparently effective in restoring a physiological dSPN/iSPN balance. However, with the progression of $\mathrm{PD}$, presynaptic and postsynaptic derangements in nigrostriatal DA transmission preclude this restoration (40), and L-DOPA pharmacotherapy now elicits both involuntary movements and motor fluctuations (41). The essential underlying mechanism would be an excessive and pulsatile stimulation of both D1 and D2 receptors (42), conceivably causing overactivity of dSPNs and underactivity of iSPNs (4). Studies in both rodent and nonhuman primate models of LID have prompted the hypothesis that overactivity of dSPNs is the actual culprit in LID (33-35). Indeed, aberrant D1 receptor-mediated signaling has 

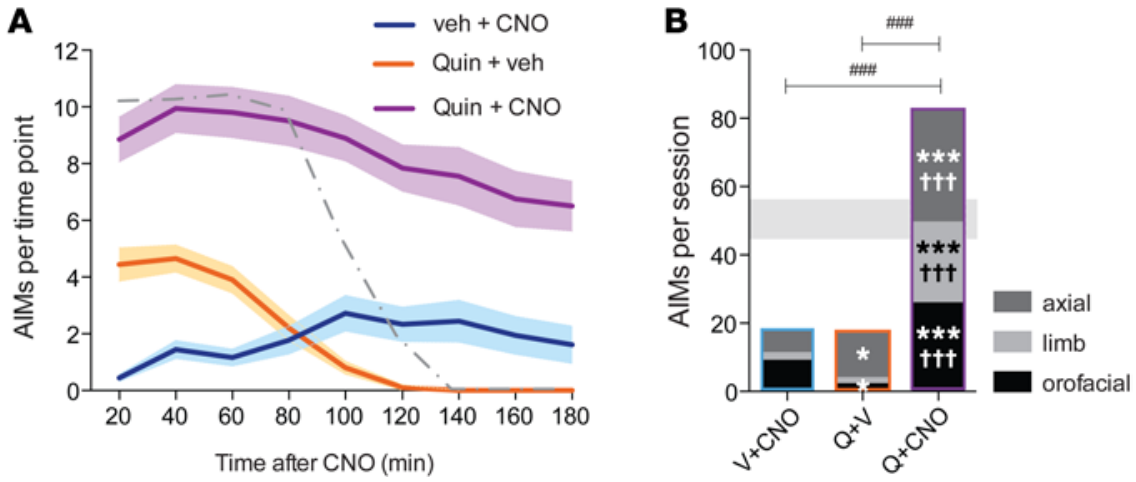

Figure 7. Combining chemogenetic dSPN-rM3Ds stimulation with D2 agonist treatment induces severe dyskinesias in 6-OHDA-lesioned mice. Ratings of axial, limb, and orofacial AIMs were performed following treatment with CNO (1 mg/kg), quinpirole (Quin) $(0.5 \mathrm{mg} / \mathrm{kg})$, or a combination of both. (A) Time course of AIMs during the test sessions (scored every 20 minutes until 180 minutes after the injection of $\mathrm{CNO}$ ). To compare dyskinesia time curves after different treatments, we introduced a dotted line representing AIMs induced by $12 \mathrm{mg} / \mathrm{kg}$ L-DOPA (which was tested in the same animals after completing the quinpirole/CNO sequence). RM 2-way ANOVA: (A) treatment, $F_{(2,26)}=46.87, P<0.001$; time, $F_{(8,208)}=$ 25.14, $P<0.001$; interaction, $F_{(16,208)}=17.41, P<0.001$. (B) Bar diagrams show the sum of AlM scores per session, where axial, limb, and orofacial AIMs are represented using different shades. The gray-shaded area represents the range (mean \pm SEM) of AIM scores induced by $12 \mathrm{mg} / \mathrm{kg}$ L-DOPA. One-way ANOVA $(n=9)$ : total AIMs: $F_{(2,23)}=13.2, P<0.001$; axial AIMS: $F_{(2,23)}=60.83, P<0.001$; limb AIMs: $F_{(2,23)}=68.80$, $P<0.001$; orofacial AlMs: $F_{(2,23)}=60.49, P<0.001$. Post hoc Tukey's test: ${ }^{\prime \prime \#} P<0.001$ for $Q+C N O$ vs. the indicated treatments. Individual AIM subtypes: ${ }^{*} P<0.05$; ${ }^{* *} P<0.001$ vs. $V+C N O ;{ }^{1 t+} P<0.001$ vs. $Q+V$.

stimulation was an asset to our study because all our behavioral assessments were based on evaluating animal-initiated movements during relatively long test sessions.

Our study of pathway-specific chemogenetic stimulation shows that activating dSPNs and iSPNs oppositely modulates whole-body motor activity in both intact and DA-denervated mice and that dSPN stimulation has stronger effects in DA-denervated mice. From this part of the study, one should conclude that a simultaneous activation of ensembles of dSPNs or iSPNs has opposite effects on an animal's general motor output, as predicted by classical models of the basal ganglia circuitry $(6,7)$ and corroborated by the results of optogenetic stimulation studies (8). Furthermore, our results show that the effect of dSPN activation is amplified in the presence of a DAdenervating lesion.

In the main part of the study, we applied pathway-specific SPN stimulation to DA-denervated mice in order to modulate both therapeutic-like and dyskinetic effects of L-DOPA treatment. Our results show that iSPN stimulation inhibits both the antiakinetic and the prodyski-

been identified as a key pathophysiological event in all current models of LID $(24,27,43,44)$. Thus, LID does not develop in parkinsonian mice that lack either the D1 receptor (24) or DA- and cAMP-regulated neuronal phosphoprotein (DARPP-32) in dSPNs (23). Moreover, the induction of dyskinesia by L-DOPA is associated with massive transcriptional changes in dSPNs, but not iSPNs (26). However, using physiological recordings and dendritic reconstruction techniques, recent studies have revealed that iSPNs undergo profound structural-functional adaptations in LID models $(38,45)$. Moreover, several studies have reported D2 agonist-induced dyskinesias in experimental models of PD (46-48). These considerations highlight the importance of testing the causality of dSPN and iSPN activity in the genesis of parkinsonian hypokinesia and dyskinesia.

This is the first study, to our knowledge, to address the above questions using pathway-specific chemogenetic stimulation. Optogenetics has been previously used to investigate the role of direct and indirect pathways in a parkinsonian mouse model (8). However, optogenetics and chemogenetics do not affect neuronal activity via similar mechanisms. In particular, optogenetic stimulation imposes spiking patterns that are independent of a neuron's afferent stimuli. Our electrophysiological data show that chemogenetic activation of SPNs does not induce any spiking per se, but that it increases the response of transduced neurons to depolarizing stimuli. This is likely to translate into an enhanced response to incoming excitatory inputs in vivo. Another fundamental difference between optogenetics and chemogenetics lies in the time frame of the effects, with the former showing rapid onset and short duration (8) while the latter exhibits slow onset and long duration (ref. 21 and our results). Along with the less invasive nature of the stimulation technique, the sustained effect of chemogenetic netic action of L-DOPA, as shown using tests of forelimb hypokinesia and AIMs ratings, respectively. In contrast, dSPN stimulation amplified the effects of L-DOPA on both forelimb hypokinesia and AIMs induction. These data indicate that dSPNs play a central role in driving both therapeutic and dyskinetic effects of DA replacement therapy in PD, verifying suggestions proposed by previous studies $(8,23)$. Somewhat unexpectedly, our results also indicate that iSPNs heavily modulate LID, a movement disorder that is currently attributed to dSPN dysregulation $(23,26)$. Thus, iSPN stimulation markedly attenuated all types of L-DOPA-induced AIMs. Moreover, combining rM3Ds-dSPN stimulation with D2 agonist treatment resulted in a synergistic prodyskinetic effect. This combined chemogeneticpharmacological intervention elicited axial, limb, and orolingual AIM scores having the same peak severity as those induced by the highest dose of L-DOPA (12 mg/kg). The present results call for a reappraisal of pathophysiological hypotheses equating LID with dSPN overactivity. Conceivably, dyskinesia induced by L-DOPA in PD could result from 2 main factors acting concomitantly, i.e., direct pathway "overactivation" via D1 receptors and indirect pathway "overinhibition" via D2 receptors. There are at least 2 mechanisms by which D2-mediated iSPN overinhibition may contribute to LID, (a) a failure to suppress competing movements when specific motor components are selected (2) and (b) a loss of the physiological lateral inhibition exerted by iSPNs onto dSPNs $(49,50)$. A model whereby both dSPNs and iSPNs contribute to LID has important translational implications that will be discussed below.

From this part of the study, we also learned that dSPN stimulation via Gq-DREADD had a therapeutic-like effect, restoring a normal use of the parkinsonian limb in the cylinder test. The restoration of forelimb use by hM3Dq-dSPN stimulation fully mimicked the beneficial effect of L-DOPA. But, unlike treatment with 
A

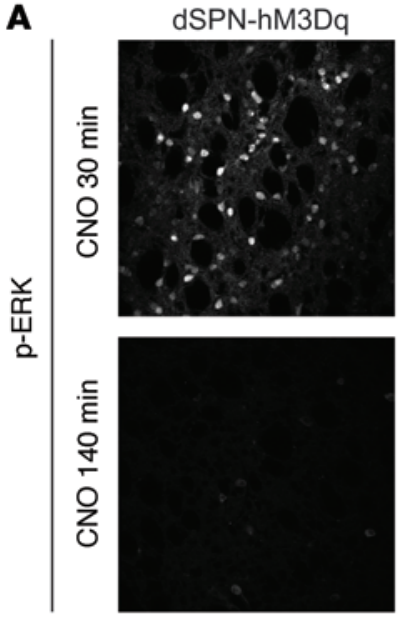

dSPN-rM3Ds

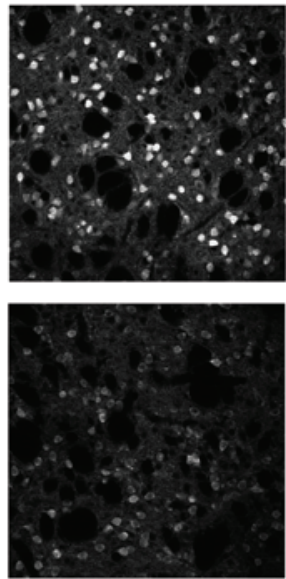

B

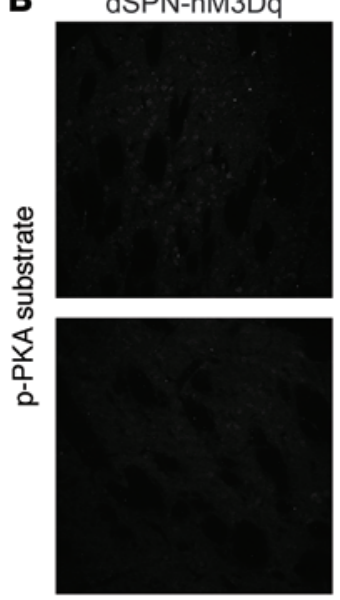

dSPN-rM3Ds
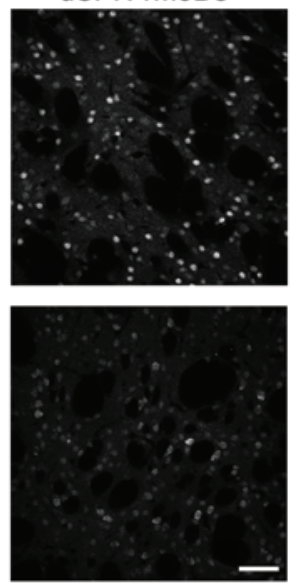

C CNO $30 \mathrm{~min} \quad \mathrm{CNO} 140 \mathrm{~min}$

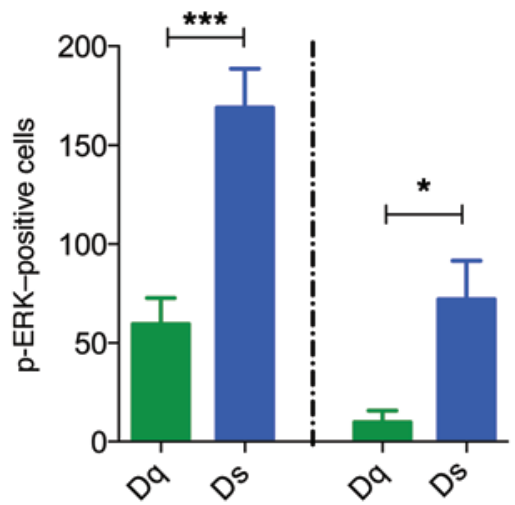

D

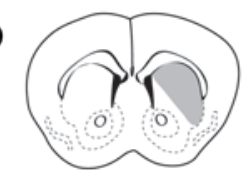

Bregma $1.18 \mathrm{~mm}$
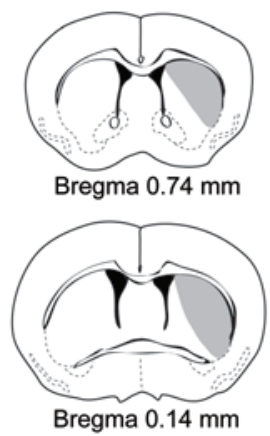

E

CNO $30 \mathrm{~min} \quad \mathrm{CNO} 140 \mathrm{~min}$

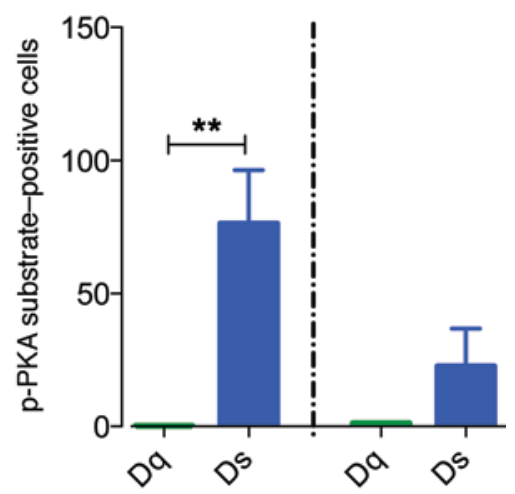

Figure 8. Activation of ERK and PKA signaling in the DA-denervated striatum upon chemogenetic stimulation of dSPNs via Gq- or Gs-DREADD. D1-Cre mice sustained 6-OHDA lesions and were transduced with hM3Dq or rM3Ds constructs. Animals were perfusion fixed at 30 minutes or 140 minutes after an injection of CNO (1 mg/kg). (A and B) Confocal photographs of striatal sections immunostained for p-ERK or p-PKA substrate, respectively. Scale bar: 100 $\mu \mathrm{m}$. (C and $\mathbf{E}$ ) Average number of p-ERK- or p-PKA substrate-positive neurons, respectively, counted within DREADD-transduced regions (represented in D, further details in Methods). Two-factor ANOVA ( $n=6-9$ per group): (C) $p$-ERK: DREADD type, $F_{(1,24)}=33.82, P<0.001$; time point, $F_{(1,24)}=24.68, P<0.001$; interaction, $F_{(1,24)}=2.579, P=0.121$. Post hoc Tukey's test: ${ }^{*} P<0.05$; ${ }^{* *} P<0.001$ vs. Gq-DREADD. (E) p-PKA substrate: DREADD type, $F_{(1,20)}=12.08, P<0.01$; time point, $F_{(1,20)}=3.541, P=0.074$; interaction, $F_{(1,20)}=3.824, P=0.065$. Post hoc Tukey's test: ${ }^{* *} P<0.01 \mathrm{vs}$. Gq-DREADD.

L-DOPA, hM3Dq-dSPN stimulation did not cause limb dyskinesia nor axial twisting movements. This is a very interesting finding, as it indicates that activating dSPNs via a Gq-linked pathway may have antiparkinsonian effects that are dissociable from the induction of involuntary movements.

In the last part of the study, we asked the question of whether chemogenetic stimulation of dSPNs is sufficient to induce AIMs in the absence of L-DOPA. Stimulation of Gq-DREADD-transduced dSPNs induced moderate orofacial AIMs, whereas axial and limb dyskinesias were virtually absent. These data suggest that orofacial AIMs have a lower induction threshold compared with limb and axial AIMs. In keeping with this interpretation, treatment of intact rodents with DA agonists can induce severe orofacial stereotypies, while trunk and limbs appear unaffected $(51,52)$. However, when DA-denervated dSPNs were stimulated via a Gs-DREADD, all types of dyskinetic movements (axial, limb, and orofacial AIMs) became manifest. Comparing molecular signaling respons- es mediated by the 2 types of DREADDs, we found that the stimulation of rM3Ds-transduced dSPNs resulted in robust activation of both ERK1/2- and PKA-linked signaling events. In contrast, the stimulation of hM3Dq-transduced dSPNs yielded only modest levels of ERK activation, lower than those induced by either L-DOPA (data not shown) or rM3Ds stimulation. These results are in line with studies on other brain regions showing that Gs- and Gq-coupled DREADD elicit distinct neuronal and behavioral outcomes (53). Our results indicate that a chemogenetic stimulation of DA-denervated dSPNs can generate involuntary movements reminiscent of LID only if it mimics signaling events that are associated with D1 receptor stimulation in the parkinsonian setting. Indeed, pharmacological stimulation of D1 receptors on intact striatal neurons activates PKA signaling with modest or no effects on ERK1/2 $(54,55)$. However, treatment of DA-denervated rodents with D1 agonists or L-DOPA results in pronounced and sustained activation of both ERK- and PKA-mediated signaling in SPNs 
A

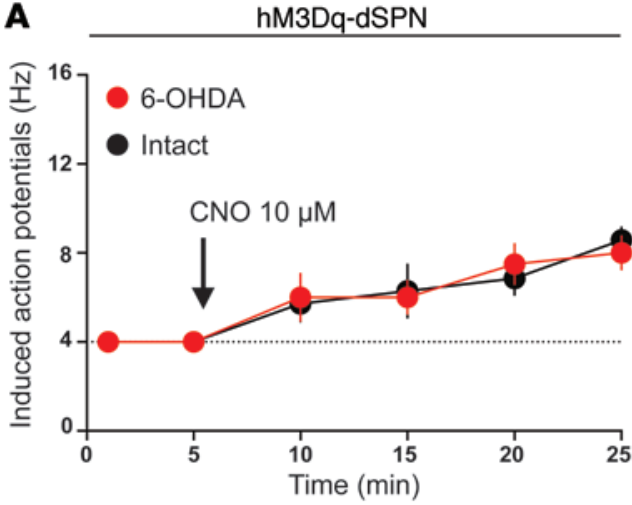

C

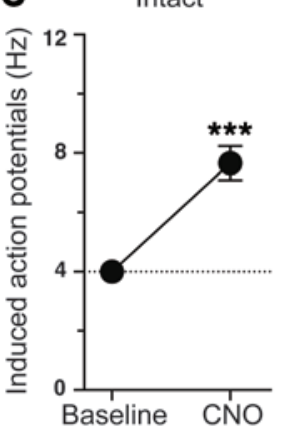

E

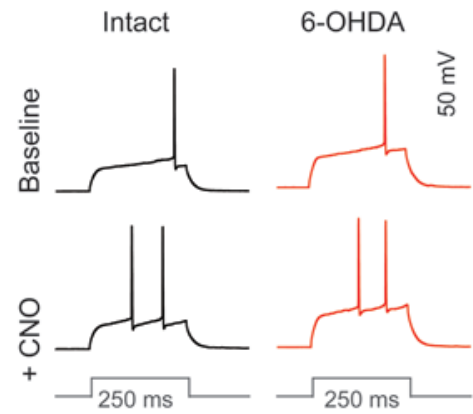

6-OHDA

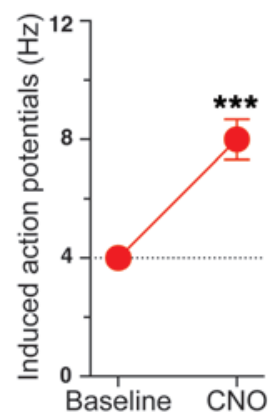

总
B

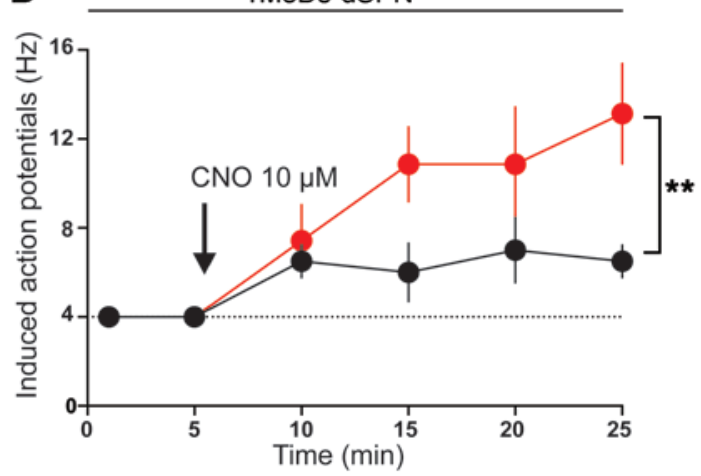

D
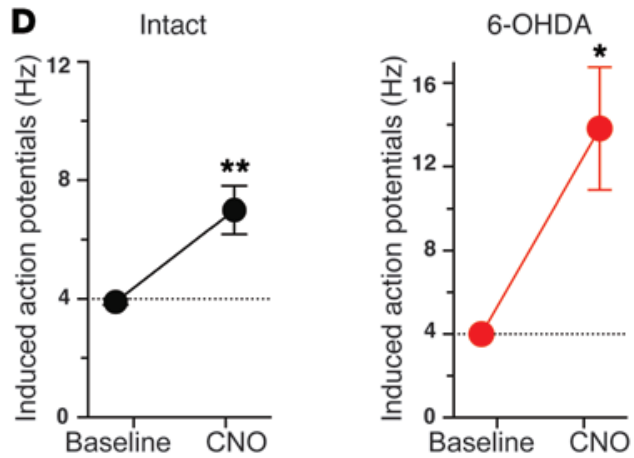

$\mathbf{F}$

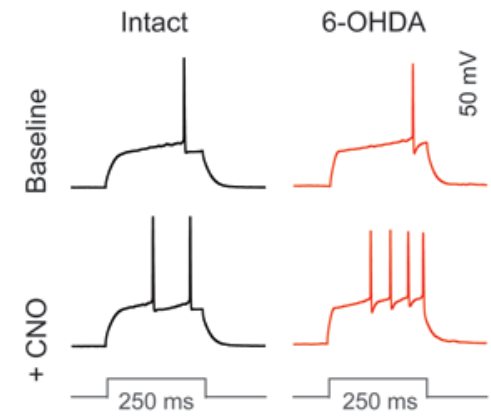

Figure 9. Electrophysiological response to CNO in DREADD-transduced dSPNs from intact and 6-OHDA-lesioned mice. Whole-cell patch clamp recordings were made in ex vivo slices from dSPNs transduced with hM3Dq (A, C, and E) or rM3Ds (B, D, and F). (A and B) Bath application of CNO $(10 \mu \mathrm{M})$ gradually increased the number of APs induced by brief somatic current pulses. No differences were observed between dSPNs of intact and 6-OHDA-lesioned mice transduced with the hM3Dq construct, while the effect of CNO stimulation was greater in the presence of a lesion in rM3Dstransduced mice. RM 2-way ANOVA ( $n=7-8$ cells per data set): $(\mathbf{A}) \mathrm{hM} 3 \mathrm{Dq}$ : effect of lesion, $F_{(1,13)}=0.001, P=0.980$; time (effect of CNO), $F_{(5,65)}=12.11$, $P<0.001$; interaction, $F_{(5,65)}=0.1870, P=0.966$. (B) rM3Ds: effect of lesion, $F_{(1,13)}=12.39, P=0.0038$; time (effect of CNO), $F_{(5,65)}=8.125, P<0.0001$; interaction, $F_{(5,65)}=1.885, P=0.109$. ${ }^{*} P<0.01$ for the effect of the lesion comparing intact vs. 6-OHDA-lesioned mice. (C and $\left.\mathbf{D}\right)$ Frequency of induced APs between baseline and CNO in intact and 6-OHDA-lesioned mice transduced with hM3Dq (C) and rM3Ds (D). Baseline and CNO refer to the average frequency of induced APs ( $\mathrm{Hz})$ during the first 5 minutes and the last 5 minutes of CNO bath application, respectively. Paired 2-tailed Student's $t$ test: ${ }^{*} P<0.05$; ${ }^{*} P<0.01$; ${ }^{* *} P<0.001$ for baseline vs. CNO. (E and $\mathbf{F}$ ) Representative traces of AP responses to current injection, at baseline and after CNO application, in hM3Dq- and rM3Ds-transduced dSPNs, respectively.

$(24,44,55)$. These signaling events are associated with abnormal potentiation of corticostriatal synaptic transmission, which is in turn linked with LID $(29,56,57)$. Moreover, striatal inhibition of either PKA or ERK signaling during L-DOPA treatment has significant antidyskinetic effects (reviewed in ref. 16). The results of chemogenetic dSPN stimulation in this study are consistent with the hypothesis that activation of both PKA- and ERK-signaling cascades in dSPNs is necessary for the emergence of dyskinesia in a PD model. Notable is the requirement of striatal DA denervation to this effect. Indeed, Gs-DREADD-mediated dSPN stimula- tion did not induce involuntary movements reminiscent of LID in intact mice. At the signal-transduction level, an upregulation of $\mathrm{G} \alpha_{\text {off }}$ (the $G$ protein that couples D1 receptors to adenylyl cyclase in the striatum) may explain the strong response to rM3Ds-dSPN stimulation in 6-OHDA-lesioned mice. Striatal upregulation of this $G$ protein has been reported in both DA-denervated animals and PD patients $(58,59)$. In line with the signaling data, the electrophysiological recordings from rM3Ds-transduced neurons showed that CNO had a more potent effect on dSPN excitability in 6-OHDA-lesioned compared with intact striata. 
Summary and translational implications. In summary, the study of pathway-specific Gq-DREADD stimulation reveals opposite roles of dSPN versus iSPN ensemble activity on horizontal and vertical movements, turning behavior, and L-DOPA-induced improvements of forelimb akinesia. Strikingly, the results also show that L-DOPA-induced axial, limb, and orofacial dyskinesias are bidirectionally modulated by dSPNs and iSPNs. These results make a strong case that neither L-DOPA-induced motor improvements nor LID are under the sole control of one pathway.

Focusing on the chemogenetic stimulation of the direct pathway, our results reveal that a behaviorally effective regimen of dSPN stimulation is not sufficient to induce dyskinesia. To induce dyskinesias reminiscent of LID, it was necessary to use DREADD construct mimicking signaling events that are downstream of D1 receptor stimulation in DA-denervated neurons. Even so, AIMs were not as severe as those induced by L-DOPA. Maximally severe AIMs were, however, reproduced by combining rM3Ds-dSPN stimulation with a D2 agonist treatment. Thus, while proving the prodyskinetic effect of a D1-mimetic signaling cascade in dSPNs, these findings also point to the importance of a concurrent change in iSPN activity.

What are the immediate translational implications of our findings? Recent pharmacological developments for the treatment of PD motor symptoms or LID have focused on identifying selective modulators of either direct or indirect pathway activity. For example, A2a receptor antagonists (60), modulators of D2-class DA receptors (61), or positive allosteric modulators of metabotropic glutamate receptor type 4 (62) have been developed to selectively reduce indirect pathway overactivity with the aim of relieving hypo/bradykinesia without inducing dyskinesia. However, none of these treatments has proven as effective as L-DOPA. Furthermore, A2a antagonists have proven to worsen LID when given as an adjunctive therapy in relatively advanced PD (reviewed in ref. 60 ). While revealing the dual control of both parkinsonian and dyskinetic features by direct and indirect pathways, our findings predict that treatments acting on both dSPNs and iSPNs will be more effective than pathway-specific interventions in improving both PD motor symptoms and LID.

\section{Methods}

Experimental subjects. The study was performed in heterozygous BAC Drd1a-Cre (D1-Cre) and Adora2a-Cre (A2a-Cre) transgenic mice (GENSAT project; founder lines EY262 and KG139, respectively, purchased at MMRRC). Mice were housed under a 12-hour light/12hour dark cycle with free access to food and water. Mice were all on a C57BL/6 genetic background and approximately 10 weeks old at the beginning of the experiments; both sexes were used.

6-OHDA lesions. Chronic striatal DA denervations were produced through unilateral injection of 6-OHDA in the medial forebrain bundle. Mice were anesthetized with a mixture of $4 \%$ isoflurane in air (Isoba vet, Apoteksbolaget) and placed in a stereotaxic frame with a mouse adaptor (Kopf Instruments). The toxin 6-OHDA hydrochloride (Sigma-Aldric) was dissolved in $0.02 \%$ ice-cold ascorbate/saline $(3.2$ $\mu \mathrm{g}$ free-base 6-OHDA/ $\mu \mathrm{l}$ ), and $0.7 \mu \mathrm{l}$ was injected at the following coordinates: anterior-posterior $=-0.7$, medial-lateral $(\mathrm{ML})=-1.2$, dorsal-ventral (DV) $=-4.7$, tooth bar: -4.0 . The injection was performed at a rate of $0.2 \mu \mathrm{l} / \mathrm{min}$ using a glass capillary attached to a $10-\mu \mathrm{l} \mathrm{Ham-}$ ilton syringe. The capillary was left in place for 2 minutes before and 4 minutes after the injection. After the surgery, the wound was closed with tissue glue and the animal received an s.c. injection of the analgesic Marcaine (bupivacaine, $2.5 \mathrm{mg} / \mathrm{ml}$, AstraZeneca; s.c.; $10 \mu \mathrm{l} / 10 \mathrm{~g}$ body weight). To prevent dehydration, mice received a s.c. injection of sterile glucose-ringer acetate $(0.6 \mathrm{ml})$ immediately after the surgery. During the first 2 to 3 weeks after surgery, mice received s.c. injections of sterile glucose-ringer acetate solution $(0.1 \mathrm{ml} / 10 \mathrm{~g}$ body weight) and dietary supplementations, as necessary. Intact mice in the study received sham-lesion surgery (capillary was inserted without delivering any injection). All 6-OHDA-lesioned animals were evaluated in the cylinder test 3 weeks after surgery, and only animals exhibiting less than $25 \%$ contralateral limb use (indicative of a successful lesion) were kept in the study. Moreover, striatal DA denervation was verified in in each animal using tyrosine hydroxylase (TH) immunohistochemistry (see Supplemental Methods). All animals included in the study had more than $80 \%$ depletion of $\mathrm{TH}$ optical density in the caudateputamen.

$A A V$ vectors and their striatal delivery. DREADD plasmids were obtained from the vector core at the University of North Carolina (Chapel Hill, North Carolina, USA) and subsequently packaged into Cre-inducible AAV vectors (serotype 5) using a double-floxed inverted open reading frame (DIO) strategy (63). The neuron-specific synapsin promoter (hSyn) was used to drive the expression of a DREADD (fused with a mCherry tag) or EGFP. Vectors were produced at the Lund AAV Vector Core (www.med.lu.se/bagadilico/technical_platforms/aav_vector_lab). The following vectors were used: AAV5-hSyn-DIO-hM3DqmCherry, AAV5-hSyn-DIO-rM3Ds-mCherry, and AAV-hSyn-DIOEGFP-WPRE (used as a control vector). The titers of the vectors were in the range of $9.9 \times 10^{13}$ to $4 \times 10^{14}$. Vector preparations were diluted 10 times and unilaterally injected into the caudate-putamen (dorsolateral striatum). Two injections ( $1 \mu \mathrm{l} \mathrm{each)} \mathrm{were} \mathrm{performed} \mathrm{at} \mathrm{the} \mathrm{following}$ coordinates: (a) anterior-posterior $=+1, \mathrm{ML}=-2.1, \mathrm{DV}=-2.9$; (b) anterior-posterior $=+0.3, \mathrm{ML}=-2.3, \mathrm{DV}=-3.0$. This injection volume ensures transduction of the entire dorsolateral striatum in the absence of nonspecific tissue damage (ref. 64 and verified in this study). The injection was performed at a rate of $0.2 \mu \mathrm{l} / \mathrm{min}$ using a glass capillary attached to a 10- $\mu$ l Hamilton syringe. The capillary was left in place for 2 minutes before and 4 minutes after the injection and then slowly retracted. For electrophysiological studies, the DREADD viral constructs were injected in combination with the EGFP control vector to allow for unequivocal identification of transduced SPNs in slices ex vivo.

To assess the extent of AAV-induced striatal transduction, striatal sections immunostained for mCherry or GFP (see below) were digitized under a $4 \times$ objective in a Nikon Eclipse 80i microscope connected to a video camera (Nikon DMX 1200F). Photomicrographs encompassing the entire mediolateral extent of the caudate-putamen were transformed to 8-bit gray scale and subsequently converted into binary pictures. Binary pictures were analyzed using Image $1.47 \mathrm{~m}$ (NIH). The percentage of striatal transduction was estimated by subtracting areas without signal from the total cross-sectional area. All animals included in the study had more than $50 \%$ transduction of the entire caudate-putamen. Transduction was never observed in the ventromedial striatum/nucleus accumbens.

Treatments with CNO, L-DOPA, and quinpirole. CNO (1/2/5 mg/ $\mathrm{kg}$, Sequoia Research Products Ltd.) was first dissolved in 0.5\% DMSO and then mixed in physiological saline solution $(9 \mathrm{~g} / \mathrm{l} \mathrm{NaCl})$. The 
same solution was used as the vehicle control. L-DOPA methyl ester (L-DOPA, 1/3/12 mg/kg; see details below) and the peripheral DOPA decarboxylase inhibitor benserazide- $\mathrm{HCl}(12 \mathrm{mg} / \mathrm{kg}$ ) (both from Sigma-Aldrich) were dissolved in physiological saline $(9 \mathrm{~g} / \mathrm{l} \mathrm{NaCl})$. When the treatments were combined, CNO was administered 20 minutes before L-DOPA. Quinpirole (Sigma-Aldrich) was given at a dose proven sufficient to exert behavioral effects via stimulation of postsynaptic D2 receptors $(65,66)$. Quinpirole $(0.5 \mathrm{mg} / \mathrm{kg})$ was dissolved in physiological saline and injected 20 minutes before CNO. All drugs (CNO, L-DOPA/benserazide, and quinpirole) were injected i.p. in a volume of $10 \mathrm{ml} / \mathrm{kg}$ body weight.

Open-field test. Whole-body movements were monitored in an open field using a video-tracking system (Stoelting ANY-MAZE video tracking) that detects the position of the animal's head, body, and tail in the arena (transparent Plexiglas boxes of $50 \times 50 \mathrm{~cm}$ ). The system provides measures of both horizontal and vertical activity. Turning movements are also detected based on the rotation of the mouse body axes and expressed as the number number of clockwise and anticlockwise full turns (here named as ipsilateral and contralateral rotations, respectively). All mice were habituated to open-field apparatus and i.p. injections for 3 consecutive days before the actual experiment. Habituation was followed by 2 consecutive days of testing, where animals were injected with CNO $(1 \mathrm{mg} / \mathrm{kg})$ or vehicle according to a randomized crossover design. Each animal was placed in the box for 60 minutes before $\mathrm{CNO}$ or vehicle injection and then recorded for an additional 120 minutes for a total test time of 3 hours. Data were collected in 5-minute bins.

Cylinder test. To assess spontaneous forelimb use, mice were placed individually inside a glass cylinder $(10 \mathrm{~cm}$ diameter, $15 \mathrm{~cm}$ height), which was located in front of 2 vertical mirrors (to see mice from all angles). Mice were placed individually in the glass cylinder and video recorded for 5 minutes, without previous habituation (67). Video recordings were used to count the number of supporting paw-to-wall contacts (fully extended digits) executed by the right or the left forelimb independently. A measure of forelimb use asymmetry was obtained by expressing the results from the limb contralateral to the lesion (left) as a percentage of the total number of wall contacts in a session.

AIMs. Three topographic subtypes of AIMs (axial, limb, orolingual, or orofacial) were rated every 20th minute during 180 minutes following drug injection $(25,68)$. Unless otherwise specified, AIM values reported in all figures are the sum of axial, limb, and orofacial scores. Axial AIMs are twisting movements or dystonic postures of neck and upper body toward the side contralateral to the lesion; limb AIMs are circular or fluttering movements of the contralateral forelimb; orofacial AIMs include twitching of facial muscles, jaw movements, and contralateral tongue protrusion. During the test, every mouse was scored on a well-characterized severity scale that is based on the duration and persistence of each dyskinetic behavior ( 0 = no dyskinesia; 1 = occasional signs of dyskinesia; 2 = frequent signs of dyskinesia, present for more than $50 \%$ of the observation time; 3 = dyskinesia present during the entire observation time, but interruptible by mild external stimuli; 4 = continuous dyskinesia, not interrupted by mild external stimuli). Before each experiment, mice were habituated to the test environment and related handling. Mice were treated with 3 escalating doses of L-DOPA $(1,3,12 \mathrm{mg} / \mathrm{kg}$ ) spanning the dose range (from very low to very high) most commonly used in this mouse model of PD. Each dose was administered daily for 6 days. Dyskinesia-rating sessions were performed on the last 2 days according to a randomized crossover design, whereby mice were injected with $\mathrm{CNO}(1 \mathrm{mg} / \mathrm{kg})$ or its vehicle $20 \mathrm{~min}$ utes before receiving L-DOPA. In experiments assessing CNO-induced AIMs, mice received daily injections of CNO for a total of 12 days (1, 2, and $5 \mathrm{mg} / \mathrm{kg} \mathrm{CNO}, 4$ days per each dose), and dyskinesia ratings were carried out on the last day of each dose. CNO-induced dyskinesias were monitored for 5 hours, although only the results from 3-hour rating sessions are reported. When combining quinpirole and CNO (1 mg/ $\mathrm{kg}$ ), the former was administered 20 minutes prior to CNO and AIMs were scored every 20th minute for 180 minutes after CNO injection.

Tissue preparation and immunohistochemistry. At 30 or 140 minutes after an i.p. injection of vehicle or CNO $(1 \mathrm{mg} / \mathrm{kg})$, mice were rapidly anesthetized with pentobarbital $(500 \mathrm{mg} / \mathrm{kg}$, i.p.; SanofiAventis) and perfused transcardially with $4 \%$ (w/v) paraformaldehyde in saline PBS, $\mathrm{pH}$ 7.4. Brains were postfixed overnight in the same solution and stored at $4^{\circ} \mathrm{C}$. Thirty micron-thick sections were cut with a Vibratome (Leica) and stored at $-20^{\circ} \mathrm{C}$ in a solution containing $30 \%(\mathrm{v} / \mathrm{v})$ ethylene glycol, $30 \%$ (v/v) glycerol, and $0.1 \mathrm{M}$ sodium phosphate buffer until they were processed for immunohistochemistry. The extent of DA denervation was verified in each animal by immunohistochemical staining for TH as described in Supplemental Methods (Immunohistochemistry) and ref. 24. The transfection efficiency of the DREADD viral constructs was verified using an antibody that recognizes the fluorescent reporter protein mCherry (anti-red fluorescent protein [anti-RFP] antibody) according to the protocol described in Supplemental Methods (Immunohistochemistry).

Double immunofluorescence was performed to visualize the expression of p-ERK or p-PKA substrate in transduced neurons. For detection of phosphorylated proteins, 0.1 M NaF was included in all buffers and incubation solutions to prevent possible dephosphorylation (for details, see immunostaining protocol described in Supplemental Methods [Immunohistochemistry]).

Quantification of phosphoprotein expression. Confocal images of mounted sections were taken on a LSM710 NLO laser-scanning microscope using a $20 \times$ objective (0.5 NA, Zeiss), applying the same adjustments for all the sections across experiments. Pictures of the transduced striatum were acquired from 3 sections representing anterior, mid, and caudal levels (represented in Figure 8D). Immunopositive neurons were counted in 2 sample areas $\left(425.1 \mu \mathrm{m}^{2}\right.$ each) per rostro-caudal level, and the results were expressed as number of positive cells per $850.2 \mu \mathrm{m}^{2}$ (cf. Figure 8, C and E). Single-plane images were used to manually count the number of neurons immunoreactive for p-ERK in a blind fashion, and Image $1.47 \mathrm{~m}(\mathrm{NIH})$ was used to automatically count the number of nuclei positive for p-PKA substrate (22).

Slice preparation for ex vivo electrophysiology. Acute striatal slices were prepared as described (38). In brief, mice were anesthetized with pentobarbital $(240 \mathrm{mg} / \mathrm{kg}$ i.p.) and shortly afterwards perfused with ice-cold artificial cerebrospinal fluid (aCSF) (see below) before decapitation. Brains were then removed, and $275-\mu \mathrm{m}$-thick, sagittal slices were cut on a vibratome (VT1200s) in aCSF containing $124 \mathrm{mM} \mathrm{NaCl}, 3 \mathrm{mM} \mathrm{KCl}, 26 \mathrm{mM} \mathrm{NaHCO}_{3}, 120 \mathrm{mM} \mathrm{NaH}_{2} \mathrm{PO}_{4}$, $2 \mathrm{mM} \mathrm{CaCl}_{2}, 1 \mathrm{mM} \mathrm{MgCl}_{2}$, and $16.66 \mathrm{mM}$ glucose. The aCSF had a molarity of typically $305 \mathrm{mOsm}$ and was at all times gassed with $\mathrm{O}_{2} / \mathrm{CO}_{2}(95 \% / 5 \%)$ to maintain $\mathrm{pH}$ and oxygenation. Brain slices were allowed to recover for at least 30 minutes at $33-34^{\circ} \mathrm{C}$. Recordings were performed at room temperature. 
Electrophysiological recordings and stimulation. Patch pipettes were pulled from thick-walled borosilicate glass on a Sutter P-90 puller; the resistance was typically 3-6 $\mathrm{mOhm}$ when filled with internal solution. The internal recording solutions contained $135.0 \mathrm{mM} \mathrm{KMeSO} 4,5.0 \mathrm{mM}$ KCL, $0.16 \mathrm{mM} \mathrm{CaCl}_{2}$, $10.0 \mathrm{mM}$ HEPES, $2.0 \mathrm{mM}$ Mg-ATP, $0.5 \mathrm{mM} \mathrm{Na}-$ GTP, $5 \mathrm{mM}$ phosphocreatine-Tris, and $5.0 \mathrm{mM}$ phosphocreatine-NA. The $\mathrm{pH}$ was adjusted to $7.25-7.30$ and osmolarity to $270-280 \mathrm{mOsm}$. For whole-cell recordings, slices were transferred to a submersion-style recording chamber mounted on a Zeiss LSM 710 NLO. Transduced cells were visually identified by the presence of EGFP and mCherry (tagged on the DREADD). After membrane rupture, cells were allowed to equilibrate for 10 to 15 minutes. Electrophysiological recordings were obtained using a Multiclamp 700B amplifier with stimulation and display obtained with pClamp (Molecular Devices). Cells were stimulated once per minute with very brief current injection $(150 \mathrm{~ms})$, with the stimulation intensity individually adjusted to elicit $1 \mathrm{AP}$. Following the establishment of the individual stimulation protocol, recordings were made for 5 minutes, with stimulation once per minute. After baseline recordings, CNO was added to the bath $(10 \mu \mathrm{M})$ and the cell recorded for another 20 minutes with ongoing stimulation (once per minute).

Statistics. Data were analyzed with GraphPad Prism software using ANOVA, factorial or by repeated measures (RM) as appropriate, and post hoc Tukey's test (factorial ANOVAs) or Bonferroni's test (RM ANOVAs), corrected for multiple comparisons. For 2-group comparisons, 2-tailed Student's $t$ test was used (paired or unpaired as appropriate). All main effects were verified using nonparametric statistics.
Study approval. All experiments were approved by the MalmöLund Ethical Committee on Animal Research, Lund District Court, Lund, Sweden.

\section{Author contributions}

$\mathrm{CA}, \mathrm{TF}$, and MAC designed research. JJ designed and produced the viral vector constructs. CA, LA, IS, and TF conducted experiments. CA, LA, IS, TF, and MAC acquired and analyzed data. CA and MAC wrote the paper with input from all coauthors.

\section{Acknowledgments}

The study was supported by grants from the Swedish Research Council (GD-2015-0006X to CA, K2012-61X-13480-13-5 and 2016-01307 to MAC); the Craaford Foundation (to CA); and the Swedish Parkinson Foundation, the Olle Engkvist Foundation, the Åhlen Foundation, the Swedish Governmental Funding for Clinical Research (ALF grant 43301), and the Basal Ganglia Disorders Linnaeus Consortium (BAGADILICO) (to MAC). The authors warmly thank Lindsay Zentveld, Ann-Christin Lindh, Elsy Ling, and Jenny Johansson for excellent technical assistance.

Address correspondence to: Cristina Alcacer or M.A. Cenci, Lund University, BMC F11, SE-221 84 Lund, Sweden. Phone: 4646.222.36.44 (C. Alcacer); or 4646.222.14.31 (M.A. Cenci); E-mail: Cristina.Alcacer@med.lu.se (C. Alcacer); or Angela.Cenci_ Nilsson@med.lu.se (M.A.Cenci).
1. Gerfen CR, Surmeier DJ. Modulation of striatal projection systems by dopamine. Annu Rev Neurosci. 2011;34:441-466.

2. Cui G, et al. Concurrent activation of striatal direct and indirect pathways during action initiation. Nature. 2013;494(7436):238-242.

3. Jin X, Tecuapetla F, Costa RM. Basal ganglia subcircuits distinctively encode the parsing and concatenation of action sequences. Nat Neurosci. 2014;17(3):423-430.

4. Surmeier DJ, Graves SM, Shen W. Dopaminergic modulation of striatal networks in health and Parkinson's disease. Curr Opin Neurobiol. 2014;29:109-117.

5. Wiecki TV, Frank MJ. Neurocomputational models of motor and cognitive deficits in Parkinson's disease. Prog Brain Res. 2010;183:275-297.

6. Albin RL, Young AB, Penney JB. The functional anatomy of basal ganglia disorders. Trends Neurosci. 1989;12(10):366-375.

7. DeLong MR. Primate models of movement disorders of basal ganglia origin. Trends Neurosci. 1990;13(7):281-285.

8. Kravitz AV, et al. Regulation of parkinsonian motor behaviours by optogenetic control of basal ganglia circuitry. Nature. 2010;466(7306):622-626.

9. Calabresi P, Picconi B, Tozzi A, Ghiglieri V, Di Filippo M. Direct and indirect pathways of basal ganglia: a critical reappraisal. Nat Neurosci. 2014;17(8):1022-1030.

10. Cazorla M, Kang UJ, Kellendonk C. Balancing the basal ganglia circuitry: a possible new role for dopamine D2 receptors in health and disease. Mov Disord. 2015;30(7):895-903.

11. Tecuapetla F, Jin X, Lima SQ, Costa RM. Comple- mentary Contributions of Striatal Projection Pathways to Action Initiation and Execution. Cell. 2016;166(3):703-715.

12. Schneider SA, Obeso JA. Clinical and pathological features of Parkinson's disease. Curr Top Behav Neurosci. 2015;22:205-220.

13. Lou JS, Kearns G, Benice T, Oken B, Sexton G, Nutt J. Levodopa improves physical fatigue in Parkinson's disease: a double-blind, placebocontrolled, crossover study. Mov Disord. 2003;18(10):1108-1114.

14. Suppa A, et al. The effect of L-dopa in Parkinson's disease as revealed by neurophysiological studies of motor sensory functions. Expert Rev Neurother. 2017;17(2):181-192.

15. Manson A, Stirpe P, Schrag A. Levodopainduced-dyskinesias clinical features, incidence, risk factors, management and impact on quality of life. J Parkinsons Dis. 2012;2(3):189-198.

16. Bastide MF, et al. Pathophysiology of L-DOPAinduced motor and non-motor complications in Parkinson's disease. Prog Neurobiol. 2015;132:96-168.

17. Calabresi P, Di Filippo M, Ghiglieri V, Tambasco N, Picconi B. Levodopa-induced dyskinesias in patients with Parkinson's disease: filling the bench-to-bedside gap. Lancet Neurol. 2010;9(11):1106-1117.

18. Gong S, et al. Targeting Cre recombinase to specific neuron populations with bacterial artificial chromosome constructs. J Neurosci. 2007;27(37):9817-9823.

19. Armbruster BN, Li X, Pausch MH, Herlitze S, Roth BL. Evolving the lock to fit the key to create a family of $G$ protein-coupled receptors potently activated by an inert ligand. Proc Natl Acad Sci US A. 2007;104(12):5163-5168.

20. Jain S, Ruiz de Azua I, Lu H, White MF, Guettier JM, Wess J. Chronic activation of a designer G(q)coupled receptor improves $\beta$ cell function. J Clin Invest. 2013;123(4):1750-1762.

21. Roth BL. DREADDs for Neuroscientists. Neuron. 2016;89(4):683-694.

22. Alcacer C, Santini E, Valjent E, Gaven F, Girault JA, Hervé D. G $\alpha$ (olf) mutation allows parsing the role of cAMP-dependent and extracellular signal-regulated kinase-dependent signaling in L-3,4-dihydroxyphenylalanine-induced dyskinesia. J Neurosci. 2012;32(17):5900-5910.

23. Bateup HS, et al. Distinct subclasses of medium spiny neurons differentially regulate striatal motor behaviors. Proc Natl Acad Sci U S A. 2010;107(33):14845-14850.

24. Darmopil S, Martín AB, De Diego IR, Ares S, Moratalla R. Genetic inactivation of dopamine D1 but not D2 receptors inhibits L-DOPAinduced dyskinesia and histone activation. Biol Psychiatry. 2009;66(6):603-613.

25. Francardo V, Recchia A, Popovic N, Andersson D, Nissbrandt H, Cenci MA. Impact of the lesion procedure on the profiles of motor impairment and molecular responsiveness to L-DOPA in the 6-hydroxydopamine mouse model of Parkinson's disease. Neurobiol Dis. 2011;42(3):327-340.

26. Heiman M, et al. Molecular adaptations of striatal spiny projection neurons during levodopainduced dyskinesia. Proc Natl Acad Sci U S A. 2014;111(12):4578-4583.

27. Santini E, et al. L-DOPA activates ERK signaling and phosphorylates histone $\mathrm{H} 3$ in the striatoni- 
gral medium spiny neurons of hemiparkinsonian mice. J Neurochem. 2009;108(3):621-633.

28. Santini E, et al. Critical involvement of cAMP/ DARPP-32 and extracellular signal-regulated protein kinase signaling in L-DOPA-induced dyskinesia. J Neurosci. 2007;27(26):6995-7005.

29. Shen W, et al. M4 muscarinic receptor signaling ameliorates striatal plasticity deficits in models of L-DOPA-induced dyskinesia. Neuron. 2016;90(5):1139.

30. Mallet N, Ballion B, Le Moine C, Gonon F. Cortical inputs and GABA interneurons imbalance projection neurons in the striatum of parkinsonian rats. J Neurosci. 2006;26(14):3875-3884.

31. Cenci MA, Whishaw IQ, Schallert T. Animal models of neurological deficits: how relevant is the rat? Nat Rev Neurosci. 2002;3(7):574-579.

32. Schallert T, Fleming SM, Leasure JL, Tillerson JL, Bland ST. CNS plasticity and assessment of forelimb sensorimotor outcome in unilateral rat models of stroke, cortical ablation, parkinsonism and spinal cord injury. Neuropharmacology. 2000;39(5):777-787.

33. Cenci MA. Dopamine dysregulation of movement control in L-DOPA-induced dyskinesia. Trends Neurosci. 2007;30(5):236-243.

34. Feyder M, Bonito-Oliva A, Fisone G. L-DOPAinduced dyskinesia and abnormal signaling in striatal medium spiny neurons: focus on dopamine d1 receptor-mediated transmission. Front Behav Neurosci. 2011;5:71.

35. Murer MG, Moratalla R. Striatal signaling in L-DOPA-induced dyskinesia: common mechanisms with drug abuse and long term memory involving $\mathrm{d} 1$ dopamine receptor stimulation. Front Neuroanat. 2011;5:51.

36. Farrell MS, et al. A Gas DREADD mouse for selective modulation of cAMP production in striatopallidal neurons. Neuropsychopharmacology. 2013;38(5):854-862.

37. Sindreu CB, Scheiner ZS, Storm DR. Ca2+-stimulated adenylyl cyclases regulate ERK-dependent activation of MSK1 during fear conditioning. Neuron. 2007;53(1):79-89.

38. Fieblinger $\mathrm{T}$, et al. Cell type-specific plasticity of striatal projection neurons in parkinsonism and L-DOPA-induced dyskinesia. Nat Commun. 2014;5:5316.

39. Planert H, Berger TK, Silberberg G. Membrane properties of striatal direct and indirect pathway neurons in mouse and rat slices and their modulation by dopamine. PLOS ONE. 2013;8(3):e57054.

40. Cenci MA. Presynaptic mechanisms of L-DOPAinduced dyskinesia: the findings, the debate, and the therapeutic implications. Front Neurol. 2014;5:242.

41. Nutt JG, Holford NH. The response to levodopa in Parkinson's disease: imposing pharmacological law and order. Ann Neurol. 1996;39(5):561-573.
42. Metman LV, Konitsiotis S, Chase TN. Pathophysiology of motor response complications in Parkinson's disease: hypotheses on the why, where, and what. Mov Disord. 2000;15(1):3-8.

43. Aubert I, et al. Increased D1 dopamine receptor signaling in levodopa-induced dyskinesia. Ann Neurol. 2005;57(1):17-26.

44. Westin JE, Vercammen L, Strome EM, Konradi C, Cenci MA. Spatiotemporal pattern of striatal ERK1/2 phosphorylation in a rat model of L-DOPA-induced dyskinesia and the role of dopamine D1 receptors. Biol Psychiatry. 2007;62(7):800-810.

45. Suárez LM, et al. L-DOPA treatment selectively restores spine density in dopamine receptor D2-expressing projection neurons in dyskinetic mice. Biol Psychiatry. 2014;75(9):711-722.

46. Bagetta V, et al. Rebalance of striatal NMDA/ AMPA receptor ratio underlies the reduced emergence of dyskinesia during D2-like dopamine agonist treatment in experimental Parkinson's disease. J Neurosci. 2012;32(49):17921-17931.

47. Bhide N, Lindenbach D, Barnum CJ, George JA, Surrena MA, Bishop C. Effects of the betaadrenergic receptor antagonist Propranolol on dyskinesia and L-DOPA-induced striatal DA efflux in the hemi-parkinsonian rat. J Neurochem. 2015;134(2):222-232.

48. Gomez-Mancilla B, Bédard PJ. Effect of chronic treatment with (+)-PHNO, a D2 agonist in MPTP-treated monkeys. Exp Neurol. 1992;117(2):185-188.

49. Dobbs LK, Kaplan AR, Lemos JC, Matsui A, Rubinstein M, Alvarez VA. Dopamine Regulation of Lateral Inhibition between Striatal Neurons Gates the Stimulant Actions of Cocaine. Neuron. 2016;90(5):1100-1113.

50. Taverna S, Ilijic E, Surmeier DJ. Recurrent collateral connections of striatal medium spiny neurons are disrupted in models of Parkinson's disease. J Neurosci. 2008;28(21):5504-5512.

51. Bez F, Francardo V, Cenci MA. Dramatic differences in susceptibility to l-DOPA-induced dyskinesia between mice that are aged before or after a nigrostriatal dopamine lesion. Neurobiol Dis. 2016;94:213-225.

52. Creese I, Iversen SD. Blockage of amphetamine induced motor stimulation and stereotypy in the adult rat following neonatal treatment with 6-hydroxydopamine. Brain Res. 1973;55(2):369-382.

53. Nakajima K, et al. Gs-coupled GPCR signalling in AgRP neurons triggers sustained increase in food intake. Nat Commun. 2016;7:10268.

54. Gerfen CR, Miyachi S, Paletzki R, Brown P. D1 dopamine receptor supersensitivity in the dopamine-depleted striatum results from a switch in the regulation of ERK1/2/MAP kinase. J Neurosci. 2002;22(12):5042-5054.

55. Fieblinger T, et al. Mechanisms of dopamine
D1 receptor-mediated ERK1/2 activation in the parkinsonian striatum and their modulation by metabotropic glutamate receptor type 5.J Neurosci. 2014;34(13):4728-4740.

56. Cerovic M, et al. Derangement of Ras-guanine nucleotide-releasing factor 1 (Ras-GRF1) and extracellular signal-regulated kinase (ERK) dependent striatal plasticity in L-DOPA-induced dyskinesia. Biol Psychiatry. 2015;77(2):106-115.

57. Picconi B, et al. Loss of bidirectional striatal synaptic plasticity in L-DOPA-induced dyskinesia. Nat Neurosci. 2003;6(5):501-506.

58. Corvol JC, et al. Persistent increase in olfactory type G-protein alpha subunit levels may underlie D1 receptor functional hypersensitivity in Parkinson disease. J Neurosci. 2004;24(31):7007-7014.

59. Marcotte ER, Sullivan RM, Mishra RK. Striatal G-proteins: effects of unilateral 6-hydroxydopamine lesions. Neurosci Lett. 1994;169(1-2):195-198.

60. Morelli M, Carta AR, Kachroo A, Schwarzschild MA. Pathophysiological roles for purines: adenosine, caffeine and urate. Prog Brain Res. 2010;183:183-208.

61. Cenci MA, Ohlin KE, Odin P. Current options and future possibilities for the treatment of dyskinesia and motor fluctuations in Parkinson's disease. CNS Neurol Disord Drug Targets. 2011;10(6):670-684.

62. Marino MJ, et al. Allosteric modulation of group III metabotropic glutamate receptor 4: a potential approach to Parkinson's disease treatment. Proc Natl Acad Sci U S A. 2003;100(23):13668-13673.

63. Atasoy D, Aponte Y, Su HH, Sternson SM. A FLEX switch targets Channelrhodopsin-2 to multiple cell types for imaging and long-range circuit mapping. J Neurosci. 2008;28(28):7025-7030.

64. Charbonnier-Beaupel F, et al. Gene expression analyses identify Narp contribution in the development of L-DOPA-induced dyskinesia. J Neurosci. 2015;35(1):96-111.

65. Frau R, Pillolla G, Bini V, Tambaro S, Devoto P, Bortolato M. Inhibition of $5 \alpha$-reductase attenuates behavioral effects of D1-, but not D2-like receptor agonists in C57BL/6 mice. Psychoneuroendocrinology. 2013;38(4):542-551.

66. Paul ML, Graybiel AM, David JC, Robertson HA. D1-like and D2-like dopamine receptors synergistically activate rotation and c-fos expression in the dopamine-depleted striatum in a rat model of Parkinson's disease. J Neurosci. 1992;12(10):3729-3742.

67. Lundblad M, Andersson M, Winkler C, Kirik D, Wierup N, Cenci MA. Pharmacological validation of behavioural measures of akinesia and dyskinesia in a rat model of Parkinson's disease. Eur JNeurosci. 2002;15(1):120-132.

68. Lundblad M, Picconi B, Lindgren H, Cenci MA. A model of L-DOPA-induced dyskinesia in 6-hydroxydopamine lesioned mice: relation to motor and cellular parameters of nigrostriatal function. Neurobiol Dis. 2004;16(1):110-123. 